\title{
TRANSVERSE CELLULAR MAPPINGS OF POLYHEDRA
}

BY

\section{ETHAN AKIN}

ABSTRACT. We generalize Marshall Cohen's notion of transverse cellular map to the polyhedral category. They are described by the following:

Proposition. Let $f: K \rightarrow L$ be a proper simplicial map of locally finite simplicial complexes. The following are equivalent:

(1) The dual cells of the map are all cones.

(2) The dual cells of the map are homogeneously collapsible in K.

(3) The inclusion of $L$ into the mapping cylinder of $f$ is collared.

(4) The mapping cylinder triad $\left(C_{f}, K, L\right)$ is homeomorphic to the product triad $(K \times I ; K \times 1, K \times 0)$ rel $K=K \times 1$.

Condition (2) is slightly weaker than $f^{-1}$ (point) is homogeneously collapsible in $K$. Condition (4) when stated more precisely implies $f$ is homotopic to a home omorphism. Furthermore, the home omorphism so defined is unique up to concordance.

The two major applications are first, to develop the proper theory of "attaching one polyhedron to another by a map of a subpolyhedron of the former into the latter". Second, we,classify when two maps from $X$ to $Y$ have homeomorphic mapping cylinder triads. This property turns out to be equivalent to the equivalence relation generated by the relation $f \sim g$, where $f, g: X \rightarrow Y$ means $f=g r$ for $r: X \rightarrow X$ some transverse cellular map.

Marshall Cohen has developed (see $\left[C_{1}\right]$ ) a theory of transverse cellular mappings defined on manifolds. They satisfy a slightly weaker condition than collapsibility of point-inverses. They are close to homeomorphisms in that they share with homeomorphisms the property that their mapping cylinder is a product. The ir interest is that they are precisely all the maps which satisfy this property.

In this paper, we generalize the notion of transverse cellularity to proper maps of locally compact polyhedra.

Proposition. Let $f: K \longrightarrow L$ be a proper, simplicial map of locally finite simplicial complexes. Then the following are equivalent:

(1) For every $A \in L$, the dual cell of $A$ with respect to $f, D(A ; f)$ (defined to be $f^{-1} D(A ; L)$ ) is homeomorphic to the cone on $\dot{D}(A ; f)$ rel $\dot{D}(A ; f)$ (where $\left.\dot{D}(A ; f) \equiv f^{-1} \dot{D}(A ; L)\right)$.

Received by the editors June 11, 1970 and, in revised form, May 4, 1971.

AMS 1969 subject classifications. Primary 5701, 5705.

Key words and phrases. Collapsing, transverse cellularity, cone, cone complex, cell-like, mapping cylinder, thickening. 
(2) For every $A \in L, D(A ; f) \searrow$ point homogeneously in $K$ (for bomogeneous collapsing see [A]).

(3) The inclusion $L \subset C_{f}\left(C_{f} \equiv\right.$ the simplicial mapping cylinder of $\left.f\right)$ is locally collared.

(4) $\left(C_{f} ; K, L\right)$ is p. l. bomeomorphic to $(K \times I ; K \times 1, K \times 0)$ rel $K=K \times 1$.

A map satisfying these is called transverse cellular.

Note that the third and fourth conditions are independent of the triangulation of $f$ because the mapping cylinder homeomorphism type is a p. 1 . invariant of the map. So it makes sense to speak of a p. l. map being transverse cellular. Also note that (4) implies $|K|$ is p. l. homeomorphic to $|L|$.

We use transverse cellularity to give the $p$. l. definition of attaching one polyhedron to another. Let $X_{0}$ be a closed subpolyhedron of a locally compact polyhedron $X$ and let $f: X_{0} \rightarrow Y_{0}$ be a proper $\mathrm{p}$. 1. map to a nother locally compact polyhedron. We define a regular extension of $f$ to be a proper p. l. map $F$ : $X \rightarrow Y$ and a closed p. l. embedding $i: Y_{0} \leftarrow Y$ such that

(i) $F^{-1}\left(i\left(Y_{0}\right)\right)=X_{0}$, and

(ii) $F \mid X_{0}=i \circ f$, and

(iii) $F: X-X_{0} \rightarrow Y-Y_{0}$ is transverse cellular.

We show that such always exist and prove the required uniqueness theorem:

Proposition. Let

$$
X \stackrel{F_{1}}{\longrightarrow} Y_{1} \stackrel{i_{1}}{\leftarrow} Y_{0} \text { and } X \stackrel{F_{2}}{\longrightarrow} Y_{2} \stackrel{i_{2}}{\longleftarrow} Y_{0}
$$

be regular extensions of $f: X_{0} \rightarrow Y_{0}$ where $X_{0} \subset X$.

There exists a regular extension $X \rightarrow{ }^{F} Y \leftarrow^{i} Y_{0}$ of $f$ and transverse cellular maps $G_{a}: Y \rightarrow Y_{a}, a=1,2$, with $G_{a}^{-1}\left(i_{\alpha}\left(Y_{0}\right)\right)=i\left(Y_{0}\right)$ and making the following diagram commute.

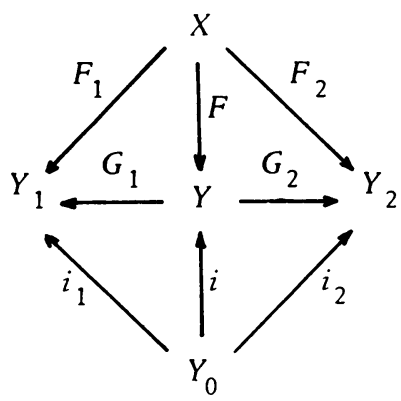

In particular, $Y_{1}$ and $Y_{2}$ are p. l. bomeomorphic.

The other major application is to a classification of mapping cylinders.

Define a relation $\approx_{e}$ between p. 1. maps. If $f_{0}: X_{0} \rightarrow Y_{0}$ and $f_{1}: X_{1} \rightarrow$ 
$Y_{1}$ then $f_{0} \approx_{e} f_{1}$ if $Y_{0}=Y_{1}$ and there exists a transverse cellular map $r$ : $X_{0} \rightarrow X_{1}$ satisfying $f_{0}=f_{1} \circ r$. $\approx_{e}$ generates an equivalence relation which we will call $\approx$.

Proposition. Let $f_{0}: X_{0} \rightarrow Y$ and $f_{1}: X_{1} \rightarrow Y$ be proper $p$. l. maps. Then the following are equivalent:

(1) $f_{0} \approx f_{1}$.

(2) There exists a p. l. homeomorphism b: $\left(C_{f_{0}}, X_{0}\right) \cong\left(C_{f_{1}}, X_{1}\right)$ rel $Y$.

Oddly enough, if one varies the range by a transverse cellular map, too, then one gets a diametrically opposed result.

Define $f_{0} \leftrightarrow{ }_{e} f_{1}$ for $f_{a}: X_{a} \rightarrow Y_{\alpha}, a=0,1$, to mean there exist transverse cellular maps $d: X_{0} \rightarrow X_{1}$ and $r: Y_{0} \rightarrow Y_{1}$ with $f_{1} \circ d=r \circ f_{0}$. Call the generated equivalence relation $\leftrightarrow$.

Proposition. $f_{0} \leftrightarrow f_{1}$ if and only if there exist bomeomorphisms $d: X_{0} \rightarrow X_{1}$ and $r: Y_{0} \rightarrow Y_{1}$ such that $f_{1}$ is bomotopic to $r \circ f_{0} \circ d^{-1}$.

I would like at this point to express my thanks to the referee, perhaps on the readers' behalf as well as my own. His detailed comments-running in length to half that of the manuscript-resulted in a complete recasting of the paper and the correction of a serious error.

I. Preliminaries. We will be looking at the piecewise linear topology of locally compact polyhedra. Unless otherwise stated all polyhedra are locally compact, subpolyhedra are closed and maps are p. 1. and proper (inverses of compact sets are compact). The corresponding simplicial category is that of locally finite simplicial complexes and proper simplicial maps. For general background as well as relations between infinite complexes and locally compact polyhedra in particular, see Hudson's book [H]. However, we will also draw heavily on the results in and the notation of Cohen's paper on regular neighborhoods $\left[\mathrm{C}_{2}\right]$ and my thesis $[\mathrm{A}]$. In particular, we will use the notation for intrinsic dimension developed in the latter. We define $d(x ; X)$ to be the intrinsic dimension of $x$ in $X ; I^{i}(X)$, the intrinsic $i$-skeleton, is $\{x \in X: d(x ; X) \leq i\}$; and in a complex $K$ a simplex $A$ is said to be a "nice face" of $B$ if $A$ is a face of $B$ and $d(x ; X)$ is constant as $x$ varies over interior $A \cup$ interior $B$.

We christen by the name "the Alexander trick" the many results which come from the fact that for compact polyhedra $X$ and $Y$ the product of the cones: $(c X) \times(c Y)$ is bomeomorpbic to the cone $c^{\prime}[(c X \times Y) \cup(X \times c Y)]$ rel $(c X \times Y) \cup$ $(X \times c Y)$ and the fact that $(c X \times Y) \cup(X \times c Y)$ is homeomorphic to the join, $X * Y$. In particular, for $Y=\{0\}, c Y$ is an interval $I$ and $s o(c X) \times I \cong$ $c^{\prime}((c X \times 0) \cup(X \times I))$ rel $(c X \times 0) \cup(X \times I)$. Also, $(c X) \times I \cong$ $c^{\prime}((c X \times i) \cup(X \times I))$, using $Y=\{0,1\}$. 
While on the subject of cones, we need to introduce the convention of the empty polyhedron 0 , satisfying cone $0=$ cone point .

In developing a category approach to mapping cylinders, we need the right category.

We introduce the category of "simplicial maps with chosen deriveds".

Objects. Simplicial maps $f: K \rightarrow L$ together with a choice of derived subdivisions on $K$ and $L$, such that $f: \eta K \rightarrow \eta L$ is also simplicial. (When $\eta K$ is a derived subdivision of $K$ and $A \in K$, we will use $\eta A$ for the point-choice in $A$ of the subdivision, e.g. the "barycenter".)

Morphisms. A morphism $G: f_{0} \rightarrow f_{1}$ where $f_{a}: K_{a} \rightarrow L_{a}, a=0,1$, is a pair of simplicial maps (written $G=\left(G_{d}, G_{r}\right)$ ) $G_{d}: K_{0} \rightarrow K_{1}$ and $G_{r}: L_{0} \rightarrow L_{1}$ also simplicial with respect to the chosen deriveds and such that $f_{1} G_{d}=G_{r} f_{0}$.

Examples. If $K$ is a complex, $K_{0}$ a subcomplex and $\eta K$ is a derived subdivision of $K$ then the identity and inclusion maps id $K: K \rightarrow K$ and inc: $K_{0} \rightarrow K$ with the derived $\eta$ chosen on domain and range are objects of this category. Furthermore, if $f: K \rightarrow L$ is an object of the category with deriveds $\eta K$ and $\eta L$, then there are natural morphisms relating the identity maps and $f$ :

$$
d_{f}=\left(\mathrm{id}_{K}, f\right): \mathrm{id}_{K} \rightarrow f, \quad r_{f}=\left(f, \mathrm{id}_{L}\right): f \rightarrow \mathrm{id}_{L} .
$$

There are also obvious functors from this category to the simplicial category: "Domain" and "Range" and on the derived level " $\eta$ Domain" and " $\eta$ Range".

II. Cone complexes.

Definition. A cone complex $G$ on a locally compact polyhedron $X$ is a locally finite covering of $X$ by compact polyhedra (called the cells of $\Xi$ ) with a boundary map $\partial$ defined on $G$, satisfying

(a) For each $\sigma \in G, \partial \sigma \subset \sigma$ and is a union of (necessarily finitely many) cells of $G$.

(b) For $\sigma, \tau$ distinct elements of $G, \stackrel{\circ}{\sigma} \cap \stackrel{\circ}{\tau}=\varnothing$ where $\stackrel{\circ}{\sigma}$ is defined to be $\sigma-\partial \sigma$.

(c) For each $\sigma \in G$, there exists a homeomorphism $\sigma \cong$ cone $\partial \sigma$ (rel $\partial \sigma$ ).

(d) If $\sigma \in \mathbb{G}$ and dimension $\sigma=0$, then and only then $\partial \sigma=0$ (the empty polyhedron).

If $c$ is weakened to $c^{\prime} . \partial \sigma$ is collared in $\sigma$, then we call $G$ a general complex on $X$.

If $c$ is strengthened to $c^{\prime \prime}$. Each $(\sigma, \partial \sigma)$ pair is a ball and boundary-sphere

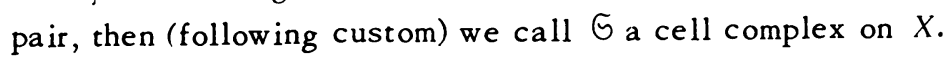

Auxiliary definitions. (1) The incidence relation $\sigma<\tau$ means $\sigma \subset \partial \tau$.

(2) If $A$ is a subset of $X$, then " $\sigma$ meets $A$ " means $\stackrel{\circ}{\sigma} \cap A \neq \varnothing$. Note that 
from (a) and (b), if $\sigma$ meets $\tau$ then $\sigma=\tau$ or $\sigma<\tau$. (Note " $\sigma$ meets $\tau$ " is not symmetric.)

(3) By (a) and (b) for each $x \in X$ there is a unique $\sigma \in \mathcal{G}$ such that $\sigma$ meets $x$, i.e. $x \in \stackrel{\circ}{\sigma}$. This $\sigma$ is called the carrier of $x$.

(4) If dimension $\sigma=0$, then we call $\sigma$ a vertex. Note that for a cone complex $\partial \sigma=0$ implies $\sigma$ is a point by (c) and our convention about the empty polyhedron.

Definition. Let $G$ be a cone complex on $X$. A choice of homeomorphism $f_{\sigma}: \sigma \cong$ cone $\partial \sigma$ rel $\partial \sigma$ is called a structuring of $\sigma$. Such a choice for each $\sigma$ in $S$ is called a structuring of $\subseteq$. $S$ together with a structuring is called a structured cone complex.

Example. If $K$ is a locally finite simplicial complex, then the dual cells of the complex form a cone complex on $|K|$ with a natural structuring given by the fact that $D(A ; K)=\eta(A) * \dot{D}(A ; K)$.

Remarks. For a cone complex there exist many different structurings and, in particular, at least one. However, by the Alexander trick any two structurings are isotopic, i.e. if $f_{\sigma}, f_{\sigma}^{\prime}: \sigma \cong$ cone $\partial \sigma$ rel $\partial \sigma$ then $f_{\sigma}$ is isotopic to $f_{\sigma}^{\prime}$. In fact, by the next remark, the isotopy of $f_{\sigma}^{\prime-1} f_{\sigma}$ to the identity on $\sigma$ rel $\partial \sigma$ extends to an ambient isotopy of $X$.

For a general complex, if $f: \sigma \cong \sigma$ rel $\partial \sigma$ and is isotopic to id $\sigma$ rel $\partial \sigma$, then the isotopy and, a fortiori $f$, extends to $X$. Just extend the isotopy up the cells by induction on dimension, noting that an isotopy of $\mathrm{id}_{\partial \tau}$ extends to an isotopy of $\mathrm{id}_{\tau}$ by the fact that $\partial \tau \subset \tau$ is collared. Thus, if $x, y \in \stackrel{\circ}{\sigma}$ are joinable by a path $x_{t}$ in $\stackrel{\circ}{\sigma}$ with $d\left(x_{t} ; \sigma\right)$ constant, then by [A, Definition II. 7] $d\left(x_{t} ; X\right)$ is constant.

Definition. If $S$ and $D$ are cone complexes on $X$ and $Y$ respectively, a map $y: \subseteq \rightarrow \mathcal{D}$ is called a cone map if it preserves incidence, i.e. $\sigma<\tau$ implies $\gamma(\sigma)<\gamma(\tau)$, and if $\gamma$ (vertex) is a vertex.

With this definition, cone-complexes and structured cone complexes become categories. We can similarly define the category of general decompositions and general decomposition maps.

Proposition 1. There is a covariant realization functor from structured cone complexes to the p. l. category defined as follows: Let $\left(\mathfrak{S}^{i},\left\{f_{\sigma}^{i}\right\}\right)$ be structured cone complexes on $X^{i}$ for $i=0,1$ and $\gamma: \mathfrak{S}^{0} \rightarrow \mathfrak{S}^{1}$ be a cone map.

Then define $\left|\left(\Im^{i},\left\{f_{\sigma}^{i}\right\}\right)\right|=X^{i}$ and $|\gamma|$ to satisfy

$$
|\gamma| \mid \sigma=\left(f_{\gamma(\sigma)}^{1}\right)^{-1} \circ(\text { cone }|\gamma| \mid \partial \sigma) \circ\left(f_{\sigma}^{0}\right) \text {. }
$$

Hence, if $\gamma: G^{0} \rightarrow G^{1}$ is a cone map, then by picking structurings we can find a map $f: X^{0} \rightarrow X^{1}$ carried by $\gamma$, i.e. $f(\stackrel{\circ}{\sigma}) \subset[\gamma(\sigma)]^{\circ}$. 
Proof. Let $|\gamma|=\gamma$ on vertices and proceed by induction up the cells of $\mathbb{G}$.

Remarks. (1) If $\gamma: G \rightarrow \supseteqq$ and we pick different structurings $\left\{f_{\sigma}\right\},\left\{f_{\sigma}^{\prime}\right\}$ on $\subseteq$ and $\left\{g_{\tau}\right\},\left\{g_{\tau}^{\prime}\right\}$ on $g$ then realizing the commutative diagram

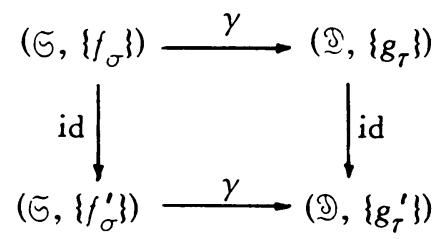

shows that the two realizations of $\gamma$ differ by homeomorphisms, which are ambiently isotopic to the identities with isotopies carried by $\subseteq$ and $\oslash$.

(2) If $g: X \rightarrow Y$ is a homeomorphism carried by an isomorphism $\gamma: \mathbb{S} \rightarrow \mathfrak{D}$, and $\left\{g_{\tau}\right\}$ is a structuring on $\mathscr{D}$ then $f_{\sigma}=(\text { cone } g \mid \partial \sigma)^{-1} \circ g_{\gamma(\sigma)} \circ g$ is a structuring on $G$, with $|\gamma|=g$.

(3) If $g: X \rightarrow Y$ is a map carried by $\gamma$ then $g$ is homotopic to $|\gamma|$ with the homotopy carried by $\gamma$.

Subcomplexes.

Definition. Let $G$ be a complex of $X$ and $X_{0}$ a subpolyhedron of $X$. "G restricts to $X_{0}$ ", or " $\subseteq$ induces a complex on $X_{0}$ ", if for each $\sigma \in G$ such that $\sigma$ meets $X_{0}, \sigma \cap X_{0} \cong$ cone $\left(\partial \sigma \cap X_{0}\right)$ rel $\partial \sigma \cap X_{0}$. Then we define $\Theta \mid X_{0}=$ $\left\{\sigma \cap X_{0}: \sigma \in G\right.$ meeting $\left.X_{0}\right\}$ with $\partial\left(\sigma \cap X_{0}\right)=(\partial \sigma) \cap X_{0}$.

(Convention. If $\partial \sigma \cap X_{0}$ is empty, we say $\partial \sigma \cap X_{0}=0$ so $\sigma \cap X_{0}$ is a point of $\stackrel{\circ}{\sigma}$, a vertex of $\left.\mathcal{S} \mid X_{0}.\right)$

If $\mathcal{G}_{\text {restricts to }} X_{0}$ and for each $\sigma$ meeting $X_{0},\left(\sigma, \sigma \cap X_{0}\right) \cong$ cone $\left(\partial \sigma, \partial \sigma \cap X_{0}\right)$ rel $\partial \sigma$ then we say $G$ induces a cone complex on the pair $\left(X, X_{0}\right)$.

Example. If $\sigma$ meets $X_{0}$ implies $\sigma \subset X_{0}$, i.e. $X_{0}$ is a union of elements of $G$ then $G$ induces a complex on $\left(X, X_{0}\right)$.

The definition of " $G$ induces a complex on $\left(X, X_{0}\right)$ "' is precisely what is needed to obtain a structuring on the pair: That is, a structuring of $G$ such that $f_{\sigma}\left(\sigma \cap X_{0}\right)=$ cone $\left(\partial \sigma \cap X_{0}\right)$ when $\sigma$ meets $X_{0}$. The analogue of Proposition 1 is true.

Similarly, $\Theta$ induces a complex on $\left(X ;\left\{X_{\alpha}\right\}\right)\left\{X_{\alpha}\right\}$ a family of subpolyhedra if for $\sigma \in \mathcal{G}$, there exists $f_{\sigma}:\left(\sigma,\left\{\sigma \cap X_{\alpha^{\prime}}\right\}\right)=$ cone $\left(\partial \sigma,\left\{\partial \sigma \cap X_{a},\right\}\right)$ rel $\partial \sigma$ where $\left\{X_{a^{\prime}}\right\}$ is the subclass consisting of those $X_{a}^{\prime}$ 's which $\sigma$ meets. Again the analogue of Proposition 1 holds.

If $\circlearrowleft$ induces a complex on $X_{0}$, then whether $G$ induces a complex on the pair is a collection of weak unknotting problems for cones: For each $\sigma$ meeting $X_{0}$, is the pair of cones $\left(\sigma, \sigma \cap X_{0}\right)$ a cone pair? For example, if $X_{0} \cap \sigma$ is a point $p$ then the question is whether $p$ can be a cone point for $\sigma$ (which 
amounts to: does it have a minimal intrinsic dimension in $\stackrel{\circ}{\sigma}$ ?). This weak unknotting question is examined in [A].

Extending structurings. In applications the following problem arises: Let $G$ be a complex on $X$ inducing a complex $G_{0}$ on $X_{0}$ and assume that we are given a structuring on $G_{0}$, when can we find a structuring on $G_{\text {which restricts to the given }}$ one on $\mathfrak{G}_{0}$ ?

Clearly, it is necessary that $G$ induce a complex on the pair $\left(X, X_{0}\right)$ but in fact more is required. This question is equivalent to a collection of unknotting problems for embedding of cones.

Let $f: c X \rightarrow c Y$ be an embedding with $f^{-1}(Y)=X$. Lickorish [L] has taught us what is means for $f$ to be unknotted: " $f$ unknots" means there exists $b: c Y \cong c Y$ rel $Y$ such that $b \circ f=c(f \mid X)$. If $X \subset Y$ and $f \mid X$ is the inclusion, then $f$ is unknotted iff there exists $g: c Y \cong c Y$ rel $Y$ extending $f$. For $b$ unknots $f$ iff $b^{-1}$ extends $f$ in this case. Thus we have

Proposition 2. Let $G$ be a cone complex on $X$, restricting to $X_{0}$ and let $\left\{f_{\sigma^{\prime}}: \sigma^{\prime} \in G \mid X_{0}\right\}$ be a structuring on $\subseteq \mid X_{0}$. Then the following are equivalent:

(1) The given structuring of $\subseteq \mid X_{0}$ extends to one of $G$, i.e. for all $\sigma \in G$ meeting $X_{0}, f\left(\sigma \cap X_{0}\right): \sigma \cap X_{0} \cong$ cone $\left(\partial \sigma \cap X_{0}\right)$ rel $\partial \sigma \cap X_{0}$ extends to a $g_{\sigma}$ : $\sigma \cong$ cone $\partial \sigma$ rel $\partial \sigma$.

(2) There exists $\left\{b_{\sigma}: \sigma \in \mathbb{S}\right\}$ a structuring of $\mathfrak{G}$, with $b_{\sigma} \circ\left(f_{\sigma \cap x_{0}}\right)^{-1}$ : cone $\left(\partial \sigma \cap X_{0}\right) \rightarrow$ cone $\partial \sigma$ is unknotted for all $\sigma$ meeting $X_{0}$.

(3) For every structuring $\left\{b_{\sigma}: \sigma \in G\right\}$ of $\subseteq, b_{\sigma} \circ\left(f_{\sigma \cap x_{0}}\right)^{-1}$ is unknotted for all $\sigma$ meeting $X_{0}$.

Remark. In applying this result we will use the criteria for unknotting developed in [A] in terms of homogeneous collapsing of sets and conewise homogeneity of the map.

III. Mapping cylinders. We now review the theory of mapping cylinders due to Marshall Cohen $\left(\left[C_{1}\right],\left[C_{3}\right]\right)$. It is perhaps worth the bother of introducing category jargon in order to note which constructions are canonical. One of the themes of this section is that many p. 1 . constructions are done by choosing a triangulation and then using a functorial simplicial construction. The choice of triangulation destroys the naturality of the construction. This is usually recovered, but only to a limited degree, by a corresponding uniqueness theorem which usually states that the construction is independent-in some sense-of the choice of triangulation. Thus, for example, "stellar neighborhood of $K_{0}$ in $K$ " (where $K_{0}$ is a subcomplex of $K$ ) is a functor on the category of pairs of simplicial complexes. The corresponding p. 1. notion of a regular neighborhood of a subpolyhedron is obtained by choosing a particular triangulation of the polyhedral 
pair $\left(X, X_{0}\right)$; namely, a first derived of one where $X_{0}$ is triangulated as a full subcomplex of $X$ (see $\left[\mathrm{C}_{2}\right.$ ], the truly enlightened approach to regular neighborhoods). The uniqueness theorem for regular neighborhoods says that the result is, up to ambient isotopy, independent of the choice of triangulation. However, naturality with respect to mappings is lost in the transition to the p. 1. category and to get it in any particular case, we choose our triangulations more carefully (i.e. to make the map in question simplicial) and use the naturality on the simplicial level. With these remarks in mind we turn to the mapping cylinder.

If $f: K \rightarrow L$ is a proper simplicial map of locally finite complexes and $\eta K$, $\eta L$ are derived subdivisions with respect to which $f$ is still simplicial, then we define the mapping cylinder of $f, C_{f}$, to be the subcomplex of $L * \eta K$ :

$$
C_{f}=\left\{A \eta B_{0} \cdots \eta B_{n}: B_{0}<B_{1}<\cdots<B_{n} \in K \text { and } f B_{0} \geq A \in L\right\} \cup L \text {. }
$$

Subdividing $L * \eta K$ to $\eta L * \eta K$ induces a subdivision $\eta C_{f}$ on the mapping cylinder:

$$
\begin{aligned}
\eta C_{f}=\left\{\eta A_{0} \cdots \eta A_{k} \eta B_{0} \cdots \eta B_{n}:\right. \\
\left.\quad A_{0}<\cdots<A_{k} \in L, A_{k} \leq f B_{0}, B_{0}<\cdots<B_{n} \in K\right\} \cup \eta L .
\end{aligned}
$$

If $K$ and $L$ are infinite then the joins are not locally finite. However, the mapping cylinders are because $f$ is proper. For let $L=\bigcup_{i=1}^{\infty} L_{i}$ with $L_{i}$ finite complexes and let $K_{i}=f^{-1} L_{i}$ (also finite because $f$ is proper). Then $K=$ $\bigcup_{i=1}^{\infty} K_{i}, C_{f}=\bigcup_{i=1}^{\infty} C_{f} \mid K_{i}$ and $\eta C_{f}=\bigcup_{i=1}^{\infty} \eta C_{f} \mid K_{i}$. If each term of the $L$-sequence is contained in the interior of the next then the $K, C_{f}$ and $\eta C_{f}$ sequences also satisfy this. But obtaining such a sequence for $C_{f}$ and $\eta C_{f}$ implies that they are locally finite.

The mapping cylinder $C_{f}$ and the subdivided mapping cylinder $\eta C_{f}$ define functors from the category of simplicial maps with chosen deriveds to the usual simplicial category. The functor applied to the morphism $G: f_{0} \rightarrow f_{1}$ written $C(G): C_{f_{0}} \rightarrow C_{f_{1}}$ or $\eta C(G): \eta C_{f_{0}} \rightarrow \eta C_{f_{1}}$ is just the restriction of $G_{r} * \eta G_{d}$ or $\eta G_{r} * \eta G_{d}$ to the subcomplex of the join. Note that if $A \leq f_{0} B$ then $G_{r} A \leq$ $G_{r} f_{0} B=f_{1} G_{d} B$ so $C(G)$ actually does map $C_{f_{0}}$ to $C_{f_{1}}$. Note that $C(G)$ and $\eta C(G)$ are proper maps, by a variation of the above union argument. Also note that $\eta C(G)$ is just the map $C(G)$ on the subdivided mapping cylinders, more precisely, they induce the same map of underlying polyhedra.

Recall the functors "Domain", "Range", " $\eta$ Domain" and " $\eta$ Range" from simplicial maps to the simplicial category. There are a host of natural transformations relating the mapping cylinder to their functors.

The retraction of the mapping cylinder onto its base $P_{f}: \quad \eta C_{f} \rightarrow \eta L$ defined by

$$
P_{f}\left(\eta A_{0} \cdots \eta A_{k} \eta B_{0} \cdots \eta B_{n}\right)=\eta A_{0} \cdots \eta A_{k} \eta f B_{0} \cdots \eta f B_{n}
$$


is a natural transformation $P_{f}: \eta C_{f} \rightarrow \eta$ Range $f$. There are also obvious inclusion transformations:

$\eta$ Domain $f \rightarrow C_{f}, \eta$ Domain $f \rightarrow \eta C_{f}$, Range $f \rightarrow C_{f}$ and $\eta$ Range $f \rightarrow \eta C_{f}$. Furthermore, $\eta$ Range $f \rightarrow \eta C_{f} \rightarrow{ }^{P} f$ Range $f$ is the identity and $\eta$ Domain $f \rightarrow$ $\eta C_{f} \rightarrow{ }^{P} f_{\eta \text { Range } f}$ is just $f$.

Finally, we recall that for two ordered simplicial complexes there is an associated triangulation of the product. In the case of $I=[0,1]$ with $0<1$ and $\eta K$ with the incidence ordering a general simplex of $\eta K \times I$ is of the form:

$$
\left(\eta A_{0}, 0\right) \ldots\left(\eta A_{k}, 0\right)\left(\eta A_{k+1}, 1\right) \ldots\left(\eta A_{n}, 1\right) \text { with } A_{0}<\ldots<A_{k} \leq A_{k+1}<\ldots<A_{n} \in K \text {. }
$$

Thus, there is a natural isomorphism: $j_{K}: \eta C_{\mathrm{id}} \cong \eta K \times I$ on the full subcategory determined by the identity maps. Note that the projection $P_{\mathrm{id}}$ is associated under this isomorphism with the projection onto the first coordinates $\pi_{K}: \eta K \times I \rightarrow \eta K$, and we get an important commutative diagram:

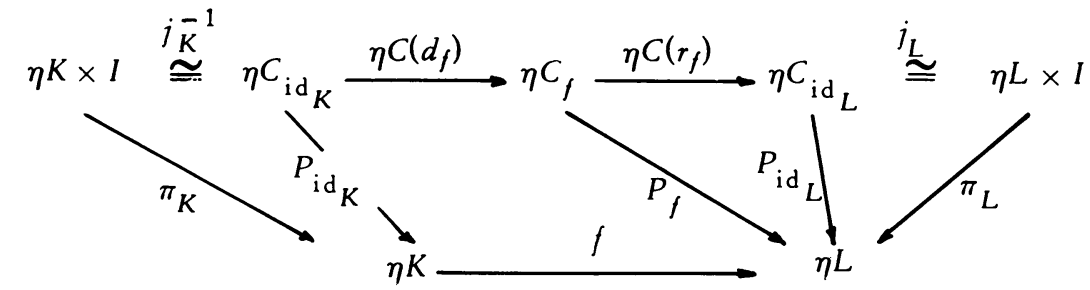

where the map across the top is $f \times l$.

It is important to know that the mapping cylinder cannot be defined functorially for the p. 1. category. This is because the obvious candidate for the mapping cylinder for $f: X \rightarrow Y$, i.e. $\bigcup\{[f(x), x] \subset Y * X: x \in X\} \cup Y$ is not, in general, a subpolyhedron of $Y * X$, e.g. if $f$ is id ${ }_{I}$. However, following the program which introduced the section we can, with Cohen, speak of a mapping cylinder of a p. 1. map, i.e. choose a triangulation of $f$ and use the simplicial mapping cylinder. Cohen's proof of the associated uniqueness theorem is an elegant application of the cell complex method and transverse cellularity.

Proposition 1. Let $f: K \rightarrow L$ be a simplicial map and $f^{*}: K^{*} \rightarrow L^{*} a$ subdivision of $f$. Choose deriveds to obtain the mapping cylinders $C_{f}$ and $C_{f} *$.

There exists a p. l. bomeomorphism $H: C_{f} \rightarrow C_{f}$, rel $K \cup L$ satisfying $H\left(C_{f} \mid K_{0}\right)=C_{f}{ }^{*} \mid K_{0}^{*}$ for all subcomplexes $K_{0}$ of $K$ (where $K_{0}^{*}$ is the subdivision of $K_{0}$ induced by $K^{*}$ ).

Proof. (See [C 1 , Proposition 9.51].)

The relation between mapping cylinders and regular neighborhoods is rather close.

If $V$ is a regular neighborhood of $L$ in $C_{f}$, where $f: K \rightarrow L$, then $(V, \dot{V})$ 
$\cong\left(C_{f}, K\right)$ rel $L\left(\left[C_{1}, 9.3\right]\right.$ and uniqueness of regular neighborhoods $)$. For the relation the other way see $\left[C_{1}, 9.7\right]$ or Proposition VI. 4.

Given a complex $L$ and a choice of derived $\eta L$, we can cut $L$ up into a cone complex using the dual cells of $L$. Cohen defines an associated structure on $K$ and $C_{f}$, where $f: K \rightarrow L$ is a simplicial map with chosen deriveds. For $A \in L$ :

$$
\begin{aligned}
& D(A ; f)=f^{-1} D(A ; L)=\left\{\eta B_{0} \cdots \eta B_{n}: A \leq f B_{0} \text { and } B_{0}<\cdots<B_{n} \in K\right\}, \\
& \dot{D}(A ; f)=f^{-1} \dot{D}(A ; L)=\left\{\eta B_{0} \cdots \eta B_{n}: A<f B_{0} \text { and } B_{0}<\cdots<B_{n} \in K\right\}, \\
& Q(A ; f)=P_{f}^{-1} D(A ; L)=\left\{\eta A_{0} \cdots \eta A_{k}: \eta B_{0} \cdots \eta B_{k} \in \eta C_{f}: A \leq A_{0}\right\}, \\
& \dot{Q}(A ; f)=P_{f}^{-1} \dot{D}(A ; L)=\left\{\eta A_{0} \cdots \eta A_{k}: \eta B_{0} \cdots \eta B_{k} \in \eta C_{f}: A<A_{0}\right\} .
\end{aligned}
$$

From the definition we obtain several important facts.

(D1) $D(A ; f)=L k\left(A ; C_{f}\right) \cap \eta K$.

(D2) Let $\bar{A}$ be a top dimensional face of $A$. Then

$$
(D, \dot{D})(A ; f)=(N, \dot{N})\left(f^{-1} \eta A ; \dot{D}(\bar{A} ; f)\right) \text {. }
$$

In particular, if $A$ is a vertex of $L$, this is

$$
(D, \dot{D})(A ; f)=(N, \dot{N})\left(f^{-1} A ; \eta K\right) \text {. }
$$

(D3) $Q(A ; f)=\eta A *(\dot{Q}(A ; f) \cup D(A ; f))$ and there is a simplicial isomorphism rel $D(A ; f)$ of $\dot{Q}(A ; f) \cup D(A ; f)$ with the subdivision of $L k\left(A ; C_{f}\right)$ induced by $\eta C_{f}$. It is defined by the identity on $D(A ; f)$ and the well-known isomorphism between $\dot{D}(A ; L)$ and $\eta L k(A ; L)$.

(D4) The decomposition is natural in the sense that $(*)$ induces the following commutative diagram for any $A \in L$.

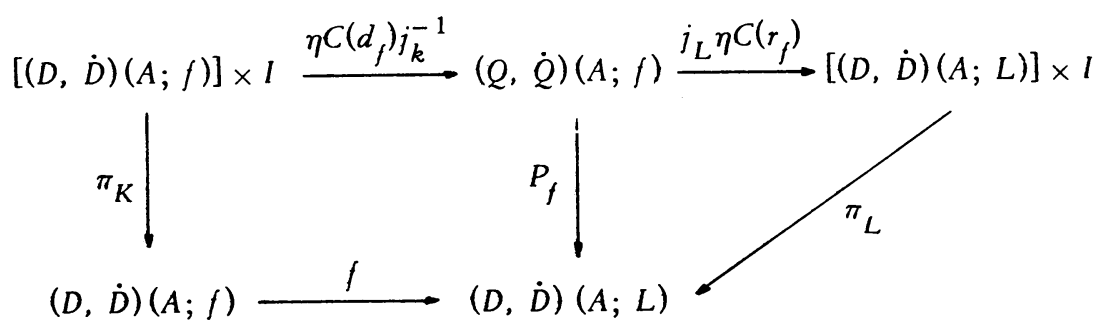

One of the crucial facts about this decomposition is that the pieces are nested regular neighborhoods $\left[C_{1}\right.$, Proposition 5.6] and $\left[C_{3}\right.$, Lemma 1.2].

Proposition 2. Let $A \in L$ and $\bar{A}$ a top-dimensional face of $A$. Then $D(A ; f)$ is a regular neighborbood of $f^{-1} \eta A$ in $\dot{D}(\bar{A} ; f)$ with boundary $\dot{D}(A ; f)$.

So in particular, if $|K|$ is a $p$. l. manifold so are all the $D(A ; f)$ 's and $\dot{D}(A ; f)$ 's. 
Remark. The canonical nature of the proof makes the relative theorem an easy variation. Thus, if $K_{0}$ is a subcomplex and $L_{0}=f\left(K_{0}\right)$ then for $A \in K_{0}$ the pair $\left(D(A ; f), D\left(A ; f \mid K_{0}\right)\right)$ is a regular neighborhood of $f^{-1} \eta A$ in $\left(\dot{D}(\bar{A} ; f), \dot{D}\left(A ; f \mid K_{0}\right)\right)$.

From this result we obtain several facts about this decomposition.

(D5) The dual cells $\{D(A ; f): A \in L\} \cup\{Q(A ; f): A \in L\}$ give a general decomposition of $\widetilde{S}_{f}\left(\overline{\left(C_{f}-L\right)}, K\right)$ (note $\overline{\left(C_{f}-L\right)}=C_{f}$ iff $f$ is onto and, in fact, $\overline{\left(C_{f}-L\right)}=C_{f}, \subset C_{f}$ where $f^{\prime}: K \rightarrow f(K)$ is the same as $\left.f\right)$. Recall that a general decomposition satisfies the axioms of a cone-complex decomposition except that $\sigma \cong$ cone $\partial \sigma$ is weakened to $\partial \sigma \subset \sigma$ is collared. The boundary is defined by $\partial D(A ; f)=\dot{D}(A ; f)$ and $\partial Q(A ; f)=\dot{Q}(A ; f) \cup D(A ; f)$.

(D6) Note that the intrinsic dimension in $\dot{D}(A ; f)$ and in $|K|$ are essentially the same. More precisely, if $\bar{A}$ is a top dimensional face of $A$, then

$$
\begin{array}{ll}
d(x ; \dot{D}(A ; f))=d(x ; \dot{D}(\bar{A} ; f))-1, & x \in \dot{D}(A ; f), \\
d(x ; D(A ; f))=d(x ; \dot{D}(\bar{A} ; f)), & x \in D(A ; f)-\dot{D}(A ; f)
\end{array}
$$

(because the boundary of a regular neighborhood is collared) and this implies by induction on $\operatorname{dim} A$,

$$
d(x ; \dot{D}(A ; f))=d(x ; K)-(\operatorname{dim} A+1) .
$$

(D7) We occasionally need the fact that the dual cells of the complex $K$ refine the dual cells of the map $f$ :

$$
D(A ; f)=\bigcup\{D(B ; K): f B=A\} .
$$

IV. Transverse cellular mappings. We now develop in detail the theory of transverse cellular mappings. This is the natural meeting point for the last two sections as this is precisely the case where the dual cell decomposition is a cone complex on the domain.

Definition. An onto simplicial map $f: K \rightarrow L$ is called transverse cellular if for each $A \in L, D(A ; f) \cong$ cone $\dot{D}(A ; \mathrm{f})$ rel $\dot{D}(A ; f)$.

Proposition 1. Let $f: K \rightarrow L$ be an onto simplicial map with deriveds chosen so that $f: \eta K \rightarrow \eta L$ is simplicial. If $f$ is transverse cellular, then there is a p. l. bomeomorphism

$$
c:\left(C_{f} ; K, L\right) \cong(L \times I ; L \times 1, L \times 0) \text { rel } L=L \times 0
$$

carried by the isomorphism of cone complexes: 


$$
\begin{aligned}
& \gamma_{f}(Q(A ; f))=D(A ; L) \times I \cong(\eta A, 0) *[\dot{D}(A ; L) \times I \cup D(A ; L) \times 1], \\
& \gamma_{f}(D(A ; f))=D(A ; L) \times 1 .
\end{aligned}
$$

Since $j_{L} \circ C\left(r_{f}\right)$ is also carried by this complex map, it is bomotopic rel $L$ to the bomeomorphism through maps carried by $\gamma_{f}$.

In particular, $f$ is bomotopic to a bomeomorphism through maps carried by the restriction of $\gamma_{f}$ taking dual cells of $f$ to those of $L$.

Proof. Note that if we use the natural structuring on $Q(A ; f)=\eta A * \partial Q(A ; f)$, then $\widetilde{\Xi}_{f}$ is a cone complex on the pair $\left(C_{f}, L\right)$ and $\widetilde{S}_{f} \mid L$ is just the dual cells of $L$ with the natural structuring. So the homeomorphism $\left|\gamma_{f}\right|$ will be the identity on $L$.

Relating cone complexes $\{D(A ; f) \times I\}$ on $K \times I$ to $\mathfrak{G}_{f}$ gives a similar theorem changing $C\left(d_{f}\right) \circ j_{K}^{-1}$ to a homeomorphism of $(K \times I ; K \times 1, K \times 0)$ to $(C ; K, L)$ rel $K \times 1=K$.

This generalizes in a straightforward way to pairs. Let $f:\left(K, K_{0}\right) \rightarrow$ $\left(L, L_{0}\right)$ be an onto simplicial map with $L_{0}=f\left(K_{0}\right)\left(K_{0}=f^{-1} L_{0}\right.$ is not necessary). Then $\widetilde{S}_{f}\left|C_{f}\right| K_{0}=\widetilde{S}_{f} \mid K_{0}$, interpreted as an equality of general complexes. Because $\left(Q(A ; f), Q\left(A ; f \mid K_{0}\right)\right)$ is always a cone pair by (D3) of $\$$ III, the only cone problems involve the dual cells.

Assume $f: K \rightarrow L$ is transverse cellular, and so $\mathscr{G}_{f}$ is a cone complex. Then $f: K_{0} \rightarrow L_{0}$ is transverse cellular iff $\mathcal{G}_{f}$ induces a cone complex on $C_{f_{0}}$. So we define " $f:\left(K, K_{0}\right) \rightarrow\left(L, L_{0}\right)$ is transverse cellular" to mean $\mathcal{E}_{f}$ induces a cone complex on the pair $\left(C_{f}, C_{f} \mid K_{0}\right)$.

If $f:\left(K, K_{0}\right) \rightarrow\left(L, L_{0}\right)$ is a transverse cellular, then by using a structuring on the complex pair, we can obtain a homeomorphism $c: C_{f} \rightarrow L \times I$ as in Proposition 1 satisfy in addition: $c\left(C_{f \mid K_{0}}\right)=L_{0} \times I$. In particular, $f$ can be homotoped to a homeomorphism as a map of pairs.

Thus going from transverse cellularity of $f: K \rightarrow L$ and $f: K_{0} \rightarrow L_{0}$ to transverse cellularity of $f:\left(K, K_{0}\right) \rightarrow\left(L, L_{0}\right)$ is equivalent to the usual collection of weak unknotting problems of whether the pair of cones $(D(A ; f)$, $\left.D\left(A ; f \mid K_{0}\right)\right)$ is a cone pair. We shall later see that if it happens that $K_{0}=$ $f^{-1}\left(L_{0}\right)$ then $f:\left(K, K_{0}\right) \rightarrow\left(L, L_{0}\right)$ is transverse cellular if $f$ is transverse cellular on each term of the pair.

Proposition 1 generalizes for families of subcomplexes. Transverse cellularity is defined by the condition that $\sigma_{f}$ induces a cone complex on the family of mapping cylinders.

There is an important sharpening of the relative case:

Proposition 2. Let $f:\left(K, K_{0}\right) \rightarrow\left(L, L_{0}\right)$ be a transverse cellular map with $f \mid K_{0}$ an isomorphism, then there is a homeomorphis $m$ 


$$
\left.c:\left(C_{f} ; K, L\right) \cong(L \times I ; L \times 1, L \times 0)\right) \text { rel } L=L \times 0
$$

carried by $\gamma_{f}$ and equal to $j_{L_{0}} \circ C\left(r_{f} \mid K_{0}\right)$ on $C_{f} \mid K_{0}$. The bomotopy of $c$ with $j_{L} \circ C\left(r_{f}\right)$ can be taken rel $C_{f} \mid K_{0} \cup L$.

Proof. In this case if $A \in L_{0}$ and $B=\left(f \mid K_{0}\right)^{-1} A$ then $D(A ; f) \cap K_{0}=$ $D\left(A ; f \mid K_{0}\right)=\eta B * \dot{D}\left(A ; f \mid K_{0}\right)$. Since $D\left(A ; f \mid K_{0}\right)$ is a subcomplex of $D(A ; f)$, the inclusion map $\eta B * \dot{D}\left(A ; f \mid K_{0}\right) \rightarrow{ }^{\text {in c }} D(A ; f)$ is conewise homogeneous (each open cone-line lies entirely in one simplex). Hence if $q$ : $\left(D(A ; f), D\left(A ; f \mid K_{0}\right)\right) \cong$ cone $\left(\dot{D}(\bar{A} ; f), \dot{D}\left(A ; f \mid K_{0}\right)\right)$ rel $\dot{D}(A ; f)$ (recall $f:\left(K, K_{0}\right) \rightarrow\left(L, L_{0}\right)$ is transverse cellular), $q \circ$ inc unknots [A, Corollary IV. 81 and hence we can find a structuring on $D(A ; f)$ which extends the natural structuring on $D\left(A ; f \mid K_{0}\right)$. Using this structuring we sharpen the relative case of Proposition 1 , because the homeomorphism we obtain of $C_{f} \mid K_{0} \cong L_{0} \times I$ by using the natural structuring is $i L_{0} \circ C\left(r_{f} \mid K_{0}\right)$.

Criteria for transverse cellularity. The definition of transverse cellularity as it stands appears rather ad hoc. Its usefulness depends on relating the definition to regular neighborhood theory to give a more directly verifiable condition than the definition.

Proposition 3. Let $f: K \rightarrow L$ be an onto simplicial map. Then the following are equivalent:

(1) $f$ is transverse cellular.

(2) For all $A \in L, D(A ; f)$ is a regular neighborbood of one of its points in $\dot{D}(\bar{A} ; f)$ where $\bar{A}$ is a top dimensional face of $A$.

(3) For all $A \in L, D(A ; f) \backslash$ point homogeneously in $K$.

Let $f:\left(K, K_{0}\right) \rightarrow\left(L, L_{0}\right)$ be an onto simplicial map of pairs such that $f:$ $K \rightarrow L$ and $f: K_{0} \rightarrow L_{0}$ are transverse cellular. Then the following are equivalent:

(1) $f\left(K, K_{0}\right) \rightarrow\left(L, L_{0}\right)$ is transverse cellular.

(2) For all $A \in L_{0},\left(D(A ; f), D\left(A ; f \mid K_{0}\right)\right)$ is a regular neighborbood of a point of $D\left(A ; f \mid K_{0}\right)$ in $\left(\dot{D}(\bar{A} ; f), \dot{D}\left(\bar{A} ; f \mid K_{0}\right)\right)$.

(3) For all $A \in L_{0}, D(A ; f)>D\left(A ; f \mid K_{0}\right)$ bomogeneously in $K$.

Proof. Recall that by Proposition III. $2, D(A ; f)$ is a regular neighborhood of $f^{-1} \eta A$ in $\dot{D}(\bar{A} ; f)$ with boundary $\dot{D}(A ; f)$. Then the following are equivalent:

(1) $D(A ; f) \cong$ cone $\dot{D}(A ; f)$ rel $\dot{D}(A ; f)$.

(2) $D(A ; f)$ is a regular neighborhood of a point in $\dot{D}(\bar{A} ; f)$.

(3) $D(A ; f)>$ point homogeneously in $\dot{D}(\bar{A} ; f)$.

(3) $\Rightarrow(2)$ by [A, III. 11d]. (2) $\Rightarrow$ (1) by uniqueness of regular neighborhoods. (1) $\Rightarrow(3)$ by collapsing down by the cone isomorphism. Homogeneity of the collapse in $\dot{D}(\bar{A} ; f)$ follows because $\dot{D}(A ; f)$ is bicollared. 
Applying (D6) of $\S$ III, we can replace in (3), "homogeneously in $\dot{D}(\bar{A} ; f)$ ", by "homogeneously in $K$ ".

In the case of pairs, (2) $\Rightarrow(1)$ is again uniqueness of regular neighborhoods. $(1) \Rightarrow(3)$ is collapsing a cone to a subcone, again using (D6) of $\$$ III to relate intrinsic dimension conditions. To obtain ( 3$) \Rightarrow(2)$, we first recall the remark that follows Proposition III.2, that the pair $D\left(A ;\left(f, f \mid K_{0}\right)\right)$ is a regular neighborhood pair of $f^{-1} \eta A$ in $\dot{D}\left(\bar{A} ;\left(f, f \mid K_{0}\right)\right)$. Then by [A, III. 11d], $D(A ; f)$ is a regular neighborhood of $D\left(A ; f \mid K_{0}\right) \bmod \dot{D}\left(A ; f \mid K_{0}\right)$ in $\dot{D}(\bar{A} ; f)$, and since $f \mid K_{0}$ is transverse cellular, $D\left(A ; f \mid K_{0}\right)$ is a regular neighborhood of a point in $\dot{D}\left(\bar{A} ; f \mid K_{0}\right)$. (2) follows from $\left[\mathrm{C}_{2}, 7.9 \mathrm{~b}\right]$.

Corollary 4. Let $f: K \rightarrow L$ be an onto simplicial map and for each $x \in L$, $f^{-1}(x) \searrow$ point homogeneously in $K$, then $f$ is transverse cellular.

Let $f:\left(K, K_{0}\right) \rightarrow\left(L, L_{0}\right)$ be an onto simplicial map with $f \mid K$ and $f \mid K_{0}$ transverse cellular and let $f^{-1}(x) \succ\left(f \mid K_{0}\right)^{-1}(x)$ bomogeneously in $K$. Then $f$ is transverse cellular as a map of pairs.

Proof. $D(A ; f)$ is a regular neighborhood of a point by [A, III.6]. For pairs, recall the remark following Proposition III. 2 and apply, analogous to the absolute case, [A, strengthened version of Theorem III.6 described on pp. 439-440].

Two results from this corollary are

Corollary 5. Let $f:\left(K, K_{0}\right) \rightarrow\left(L, L_{0}\right)$ be a simplicial map with $f: K \rightarrow L$ and $f: K_{0} \rightarrow L_{0}$ transverse cellular and assume $K_{0}=f^{-1} L_{0}$. Then $f:\left(K, K_{0}\right)$ $\rightarrow\left(L, L_{0}\right)$ is transverse cellular.

Corollary 6. Let $f:(B, S) \rightarrow(X, Y)$ be an onto p. l. map with $f^{-1} Y=S$ and such that $B$ is a $p$. l. ball with boundary $S$ and sucb that for $x \in X, f^{-1}(x)$ is collapsible. Then any triangulation of $f$ is transverse cellular and bence $X$ is a p. l. ball bomeomorphic to $B$ with boundary $Y$.

Because of Corollary 4, we ask the following: $X_{0} \searrow$ point homogeneously in $X$, and $p \in X_{0}$; then does $X_{0} \searrow p$ homogeneously in $X$ ?

Lemma 7. Let $X_{0} \subset X$ and assume $X_{0}$ collapses to a point bomogeneously in $X$. Then for $p \in X_{0}, X_{0} \searrow_{b} p$ in $X$ iff $d(p ; X)=\min \left\{d(x ; X): x \in X_{0}\right\}$.

Proof. Define $m\left(X_{0} ; X\right)=\min \left\{d(x ; X): x \in X_{0}\right\}$. Then if $X_{0} \searrow_{b} X_{1}$ in $X$ then $m\left(X_{0} ; X\right)=m\left(X_{1} ; X\right)$, for it is easily seen to be true for elementary geometrical homogeneous collapses. Hence, if $X_{0} \searrow_{b} p$ in $X$, then $m\left(X_{0} ; X\right)=$ $m(p ; X)=d(p ; X)$.

Conversely, if $m=m\left(X_{0} ; X\right)$ then since $X_{0} \searrow$ pt homogeneously and since [A, III. 10$]$ this can be done in order of decreasing intrinsic dimensions, $X_{0} \searrow_{b} X_{0} \cap I^{m}(X)=X_{0} \cap\left(I^{m}(X)-I^{m-1}(X)\right) \succ$ pt in $X$. Now if $d(p ; X)=$ 
$m\left(X_{0} ; X\right)$ then $p \in X_{0} \cap I^{m}(X)$ which is collapsible and hence $X_{0} \cap$ $I^{m}(X) \searrow p$. Since $X_{0} \cap I^{m}(X)=X_{0} \cap\left(I^{m}(X)-I^{m-1}(X)\right)(m$ is minimal $)$, this collapse is homogeneous in $X$.

Corollary 8. Let $f: K \rightarrow L$ be an onto simplicial map with $f^{-1}(x)>$ point bomogeneously in $K$ for each $x \in L$ and let $K_{0} \subset K$ sucb that $f \mid K_{0}$ is a bomeomorphism onto $f\left(K_{0}\right)=L_{0}$. Then if for each $y \in K_{0}, d(y ; K)=$ $\min \left\{d(z ; K): z \in f^{-1} f(y)\right\}$, then $f:\left(K, K_{0}\right) \rightarrow\left(L, L_{0}\right)$ is transverse cellular.

Proof. Lemma 7 and Corollary 4.

The necessity for homogeneous collapsing is illustrated by the map from the disc shown below so that $f^{-1}(\bar{a})=[a, b]$, so $f \mid b$ is an isomorphism,
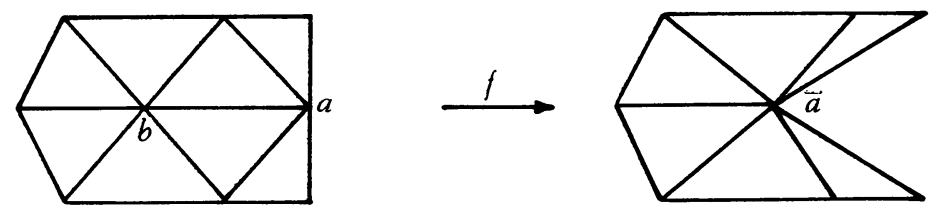

but clearly no homeomorphism of balls will take the interior point $b$ to the boundary point $\bar{a}$. Note that the collapse $[a, b] \searrow b$ is not homogeneous.

We now show that despite its simplicial definition, transverse cellularity is a p. 1. phenomenon. To do this, we use the fact that the simplicial mapping cylinder is a p. I. invariant and then follow Cohen by proving a strong converse to Proposition 1.

Proposition 9 (closed). Let $f: K \rightarrow L$ be a simplicial mapping and $Y$ a subpolybedron of $L$, then $P_{f}^{-1}(Y)$ is $p$. l. bomeomorpbic to a mapping cylinder for $f \mid f^{-1}(Y)$ rel $f^{-1}(Y) \cup Y$.

Proof. Let $P_{f}^{-1}(Y) \cap K=f^{-1}(Y)=X$. Triangulate $f \mid X$ to obtain $\hat{f}: K_{0} \rightarrow$ $L_{0}$ simplicial with $K_{0}$ (resp. $L_{0}$ ) a refinement of $K$ (resp. $L$ ). Note that we do not assume that $K_{0}$ is obtained from a subdivision of $K$.

We define isomorphic cell complexes on $P_{f}^{-1}\left(L_{0}\right)$ and $C \hat{f} \mid K_{0}$ whose realization will give the desired homeomorphism.

Let $\sigma \in L_{0}$, with $A \in L$ the carrier of $\sigma$ and let $B \in K$ be such that $f B=A$. That the complexes exist follows from the sublemma:

Sublemma. (1) $C_{\hat{f} \mid f^{-1}(\sigma) \cap B}$ is a $\operatorname{dim}\left(f^{-1}(\sigma) \cap B\right)+1$ p. l. ball with boundary $=C_{\hat{f} \mid f^{-1}(\dot{\sigma}) \cap B} \cup C_{\hat{f} \mid f^{-1}(\sigma) \cap \dot{B}} \cup\left(f^{-1}(\sigma) \cap B\right) \cup \sigma$.

(2) $P_{f}^{-1}(\sigma) \cap C_{f \mid B}$ is a $\operatorname{dim}\left(f^{-1}(\sigma) \cap B\right)+1$ p. l. ball with boundary $=\left(P_{f}^{-1}(\dot{\sigma}) \cap C_{f \mid B}\right) \cup\left(P_{f}^{-1}(\sigma) \cap C_{f \mid \dot{B}}\right) \cup\left(f^{-1}(\sigma) \cap B\right) \cup \sigma$.

Assuming the sublemma we compare the following complexes by the obvious isomorphism and obtain the homeomorphism: 


$$
\begin{aligned}
&\left\{P_{f}^{-1}(\sigma) \cap C_{f \mid B}, f^{-1}(\sigma) \cap B, \sigma: \quad \sigma \in L_{0} \text { and } f B=\operatorname{Car}(\sigma) \text { in } L\right\} \\
&\left(\text { decomposing }\left(P_{f}^{-1}\left(L_{0}\right) ; K_{0}, L_{0}\right)\right) . \\
&\left\{C_{\hat{f} \mid f^{-1}(\sigma) \cap B}, f^{-1}(\sigma) \cap B, \sigma: \sigma \in L_{0} \text { and } f B=\operatorname{Car}(\sigma) \text { in } L\right\}\left(\text { decomposing }\left(C \hat{f} \mid K_{0} ; K_{0}, L_{0}\right)\right) .
\end{aligned}
$$

The key to proving the sublemma was given in the proof of $\left[C_{1}, 9.5\right]$. In fact, since $f: f^{-1}(\sigma) \cap B \rightarrow \sigma$ is an onto linear map of a convex cell, for the proof of part 1, we need only note that $\partial\left(f^{-1}(\sigma) \cap B\right)=\left(f^{-1}(\dot{\sigma}) \cap B\right) \cup\left(f^{-1}(\sigma) \cap \dot{B}\right)$, and

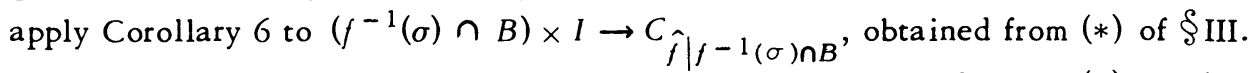

To prove the second part, we again use the commutative diagram $(*)$ which restricts to the following:

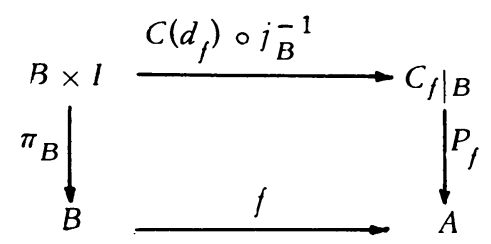

Hence, $C\left(d_{f}\right) \circ j_{B}^{-1}$ restricts to a map:

$$
\begin{aligned}
& \left(\left(f^{-1}(\sigma) \cap B\right) \times I ;\left(f^{-1}(\dot{\sigma}) \cap B\right) \times I,\left(f^{-1}(\sigma) \cap \dot{B}\right) \times I,\right. \\
& \left.\quad\left(f^{-1}(\sigma) \cap B\right) \times 1,\left(f^{-1}(\sigma) \cap B\right) \times 0\right) \\
& \rightarrow\left(P_{f}^{-1}(\sigma) \cap C_{f \mid B} ; P_{f}^{-1}(\dot{\sigma}) \cap C_{f \mid B}, P_{f}^{-1}(\sigma) \cap C_{f \mid \dot{B}}, f^{-1}(\sigma) \cap B, \sigma\right) .
\end{aligned}
$$

Furthermore, the inverse of each point is collapsible by $\left[C_{1}, 3.3\right]$ or see $\$ \mathrm{VI}$. The result follows from Corollary 6 .

Proposition 9 (open). Let $f: K \rightarrow L$ be a simplicial mapping and $U$ an open subset of $L$, then $P^{-1}(U)$ is $p$. l. homeomorpbic to a mapping cylinder for $f \mid f^{-1}(U)$ rel $U \cup f^{-1}(U)$.

Proof. Triangulate with refinements of $K$ and $L$ to obtain $\hat{f}: f^{-1}(U) \rightarrow U$ and a mapping cylinder $C_{\hat{f}}$. We want to obtain a homeomorphism of $P_{f}^{-1}(U)$ with $C_{\hat{f}}$. To do this we use the Morton Brown infinite union swindle and repeatedly apply Propositon 9 (closed).

Let $U=\bigcup_{i=1}^{\infty} V_{i},\left\{V_{i}\right\}$ an increasing sequence of finite subcomplexes of $U$. Since each $V_{i}$ is compact, Proposition 9 (closed) applies and we have, for each $i, k_{i}: P_{f}^{-1}\left(V_{i}\right) \cong P_{\hat{f}}^{-1}\left(V_{i}\right)=C_{\hat{f} \mid f^{-1}\left(V_{i}\right)}$ rel $V_{i} \cup f^{-1}\left(V_{i}\right)$. We refer to the proof of Proposition 9 (closed) to show that we can assume $k_{i+1} \mid P_{f}^{-1}\left(V_{i}\right)=k_{i}$. Then $\bigcup_{i=1}^{\infty} k_{i}: P_{f}^{-1}(U) \cong C_{\hat{f}}$. To refine the proof of Proposition 9 (closed) note that the cell-complexes constructed for $P_{f}^{-1}\left(V_{i+1}\right)$ and $C_{\hat{f} \mid f-1}\left(V_{i+1}\right)$ have as subcom- 
plexes respectively the cell complexes for $P_{f}^{-1}\left(V_{i}\right)$ and $C_{\hat{f} \mid f-1}\left(V_{i}\right)$. So if $k_{i}: P_{f}^{-1}\left(V_{i}\right) \rightarrow C_{\hat{f} \mid f-1}\left(V_{i}\right)$ has been defined preserving this complex isomorphism, $k_{i+1}$ can be realized to extend $k_{i}$.

Now we obtain a p. 1. invariant criterion for transverse cellularity.

Proposition 10. If $f: K \rightarrow L$ is a simplicial map, with $L_{0}$ a subcomplex of $L$ and $K_{0}=f^{-1} L_{0}$, then the following are equivalent.

(1) $D(A ; f)$ is a cone on $\dot{D}(A ; f)$ for all $A \in L-L_{0}$.

(2) $C_{f}$ is locally collared on $L-L_{0}$.

(3) For any choice of triangulations, $f:\left|K-K_{0}\right| \rightarrow\left|L-L_{0}\right|$ is transverse cellular.

(4) There exists a triangulation of $f:\left|K-K_{0}\right| \rightarrow\left|L-L_{0}\right|$ which is trans. verse cellular.

Proof. (2) $\Rightarrow$ (1) by induction on $\operatorname{dim} L$.

By local collaring, we have for $A \in L-L_{0}$ the isomorph of its link-recall property (D3) of $\S \mathrm{III}-$ is a cone, i.e. $\dot{Q}(A ; f) \cup D(A ; f) \cong$ cone $\dot{D}(A ; L)$ rel $\dot{D}(A ; L)$, implying in particular $D(A ; f) \neq \varnothing$ so $f$ is onto $L-L_{0}$.

Since $\dot{Q}(A ; f)=P_{f}^{-1} \dot{D}(A ; L)$ it follows from Proposition 9 (closed) that $(\dot{Q}(A ; f) ; \dot{D}(A ; f), \dot{D}(A ; L))$ is homeomorphic to a mapping cylinder of $f \mid \dot{D}(A ; f)$. Hence, by inductive hypothesis $f \mid \dot{D}(A ; f)$ is transverse cellular (since $\dot{D}(A ; L) \subset \dot{Q}(A ; f)$ is collared) and so by Proposition $1,(\dot{Q}(A ; f) ; \dot{D}(A ; f), \dot{D}(A ; L))$ is homeomorphic to $(\dot{D}(A ; f) \times I ; \dot{D}(A ; f) \times 0, \dot{D}(A ; f) \times 1)$. Hence the cone $\dot{Q}(A ; f)$ $\cup D(A ; f)$ is just $D(A ; f)$ with an exterior collar. So $D(A ; f) \cong \dot{Q}(A ; f) \cup$ $D(A ; f)$ and is hence a cone.

$(1) \Rightarrow(2)$. If $D(A ; f)$ is a cone for all $A \in L-L_{0}$ then $\gamma_{f}$ gives a cone complex isomorphism between $\dot{Q}(A ; f) \cup D(A ; f)$ and $(\dot{D}(A ; L) \times I) \cup$ $(D(A ; L) \times 1)$ indexed by $\{B: A<B\}$. So $L k\left(A ; C_{f}\right)$ is a cone by $\S I I I(D 3)$. Essentially, we just reverse the above argument.

To relate these to (3) and (4), note that by Proposition 9 (open) $P_{f}^{-1}\left(L-L_{0}\right)$ is homeomorphic rel $\left|K-K_{0}\right| \cup\left|L-L_{0}\right|$ to any mapping cylinder for $f:\left|K-K_{0}\right| \rightarrow\left|L-L_{0}\right|$. Hence, (4) $\Rightarrow$ (2) a fortiori by Proposition 1.

Finally, given (2), let $\hat{f}$ be any triangulation of $f|| K-K_{0} \mid$. By Proposition 9 (open) again, $P_{f}^{-1}\left(L-L_{0}\right)$ is homeomorphic to $C_{\hat{f}}$ and hence $\left|L-L_{0}\right| C$ $C_{\hat{f}}$ is collared. Now $(2) \Rightarrow(1)$ applied to the absolute case of $\hat{f}$ gives $\hat{f}$ transverse cellular. Thus $(2) \Rightarrow(3)$.

Clearly, (3) $\Rightarrow(4)$.

Remark. From the proof of $(2) \Rightarrow(1)$ we obtain a useful fact about transverse cellularity on the dual cells. Let $\dot{f}: K \rightarrow L$ be simplicial and, $A \in L$ and $L k\left(A ; C_{f}\right) \cong$ cone $L k(A ; L)$ rel $L k(A ; L) ;$ then 
(1) $f \mid \dot{D}(A ; f)$ is transverse cellular.

(2) $D(A ; f)$ is a cone on $\dot{D}(A ; f)$.

(3) $f \mid D(A ; f)$ is transverse cellular.

(1) and (2) are directly contained in the above proof. From it we obtain an isomorphism

$$
\begin{aligned}
(\dot{Q}(A ; f) \cup & D(A ; f) ; D(A ; f), \dot{D}(A ; L)) \\
& \cong((\dot{D}(A ; f) \times I) \cup(D(A ; f) \times 1) ; D(A ; f) \times 1, \dot{D}(A ; f) \times 0) ;
\end{aligned}
$$

coning this and using the Alexander trick, we get a homeomorphism

$$
(Q(A ; f) ; D(A ; f), D(A ; L)) \cong(D(A ; f) \times I ; D(A ; f) \times 1, D(A ; f) \times 0) .
$$

Since $Q(A ; f)$ is by Proposition 9 a mapping cylinder for $f \mid D(A ; f)$, Proposition 10 implies that $f \mid D(A ; f)$ is transverse cellular.

We have used the invariance of the mapping cylinder and of the property of local collaring to obtain invariance of transverse cellularity. Note that the logic of this argument depended on the obvious p. 1. invariance of the property $f^{-1}(x) \searrow$ point homogeneously, which is stronger than transverse cellularity. The dependence comes from repeated applications of Corollary 6, in Proposition 9 for example.

From Corollary 10, we can define a p. 1. map $/: X \rightarrow Y$ to be transverse cellular if it is so for any triangulation.

Quasi-concordance and quasi-isotopy. We also obtain a method for making constructions of transverse cellular mappings. Recall that a general decomposition of a space is a collection of pieces that fit together like a cone complex but $\partial \sigma$ is only collared in $\sigma$ instead of $\sigma \cong$ cone $\partial \sigma$. An isomorphism of general decompositions is a bijection preserving incidence in each direction.

Proposition 11. Let $f: X \rightarrow Y$ be an onto $p$. l. map carried by an isomorphism $\gamma: \mathfrak{G} \rightarrow$ D with $\mathcal{G}$, I general complexes on $X$ and $Y$ respectively and $f^{-1}(\partial \gamma \sigma)=\partial \sigma$. Assume that $f: \sigma \rightarrow \gamma(\sigma)$ is transverse cellular for each $\sigma \in \mathfrak{G}$. Then $f$ is transverse cellular.

Proof. Proceed by induction on the dimension of cells in $\mathfrak{G}$. Assume $\operatorname{dim} X=n$ and that there is only one $n$-dimensional cell. Let $\sigma$ be the top dimensional cell of $\subseteq$ and $X_{\sigma}=\mathrm{Cl}(X-\sigma)=\mathbf{U}\left(\Xi_{-} \sigma\right)$, and $Y_{\sigma}=\mathrm{Cl}(Y-\gamma \sigma)=$ $\cup(D-\gamma \sigma)$. By inductive hypothesis $f: X_{\sigma} \rightarrow Y_{\sigma}$ and $f: \partial \sigma \rightarrow \gamma \partial \sigma=\partial y \sigma$ are transverse cellular. Since $f^{-1} \gamma \partial \sigma=\partial \sigma, f:(\sigma, \partial \sigma) \rightarrow(y \sigma, \partial \gamma \sigma)$ and $f:$ $\left(X_{\sigma}, \partial \sigma\right) \longrightarrow\left(X_{\gamma \sigma}, \partial \gamma \sigma\right)$ are transverse cellular by Corollary 5 . Triangulating so that everything is subcomplex, we obtain isomorphisms

$$
\begin{array}{cc}
j_{1}:\left(C_{f \mid x_{\sigma}}, C_{f \mid \partial \sigma}\right) \cong\left(Y_{\sigma}, \gamma \partial \sigma\right) \times I \text { rel } Y_{\sigma}=Y_{\sigma} \times 0, \\
j_{2}:\left(C_{f \mid \sigma}, C_{f \mid \partial \sigma}\right) \cong(\gamma \sigma, \gamma \partial \sigma) \times I \text { rel } \gamma \sigma=\gamma \sigma \times 0 .
\end{array}
$$


Since $\partial \sigma \subset \sigma$ is collared, we can change $j_{2}$ to agree with $j_{1}$ on $C_{f} \mid \partial \sigma$. (Extend the concordance of id $\gamma \partial \sigma$ given by $j_{1} j_{2}^{-1}$ to a concordance of id ${ }_{\gamma \sigma}$ and compose with $j_{2}$. Note that if $A$ is compact and $B \subset A$ is collared then any concordance of $\mathrm{id}_{B}$ extends to one of $\mathrm{id}_{A}$ because $(A \times I ; A \times 0 \cup B \times I, A \times 1) \cong$ $(A \times I ; A \times 0, A \times 1 \cup B \times I)$.) Glue them together to obtain $j: C_{f} \cong Y \times I$ rel $Y$ $=Y \times 0$ and hence $f$ is transverse cellular by Proposition 10 .

In general, just deal with all of the $n$-dimensional cells at the same time as above.

Remarks. Alternative to the assumption $f^{-1}(\partial \gamma \sigma)=\partial \sigma$, we can just assume $f:(\sigma, \partial \sigma) \rightarrow(\gamma \sigma, \partial \gamma \sigma)$ is transverse cellular.

For a converse to this theorem, see Proposition V.9.

Corollary 12. Let $f: X \rightarrow Y, g: Y \rightarrow Z$ be transverse cellular p. l. maps. Then $g f$ is transverse cellular.

Proof. Triangulate to obtain $K \rightarrow^{f} L \rightarrow^{8} M$ simplicial. Since $g$ is transverse cellular, we have $D(A ; g)=$ cone $\dot{D}(A ; g)$ for $A \in M$. By Remark 3 after Proposition $10, f \mid D(B ; f)$ for each $B$ in $L$ is transverse cellular. $D(A ; g)$ and $\dot{D}(A ; g)$ for each $A$ in $M$ is a union of dual cells of $L$ by (D7) of $\S I I I$ and hence $D(A ; g f)=f^{-1} D(A ; g)$ is a union of dual cells of $f$. So $f:(D(A ; g f)$, $\dot{D}(A ; g f)) \rightarrow(D(A ; g), \dot{D}(A ; g))$ is transverse cellular by the above proposition and Corollary 5. Since $(D(A ; g), \dot{D}(A ; g))$ is a cone pair, $(D(A ; g f), \dot{D}(A ; g f))$ is by Proposition 1.

In applying Proposition 11 to cone complexes to make inductive construction, we will need the following:

Lemma 13. Let $f: X \rightarrow Y$ be a transverse cellular map. Then $c f:(c X, X)$ $\rightarrow(c Y, Y)$ (the cone of $f)$ is transverse cellular.

Proof. By Corollary 5, we need only show $c f: c X \rightarrow c Y$ is transverse cellular. Triangulate so that $f: K \rightarrow L$ is simplicial. Choose deriveds so that $f: \eta c K \rightarrow \eta c L$ is simplicial. $f^{-1}(L)=K$ so for $A \in L, D(A ; c f) \searrow$ $D(A ; f) \searrow$ point homogeneously. $(c f)^{-1}(c)=c$ so $D(c ; c f) \searrow(c f)^{-1} c=c$ homogeneously.

Consider the simplicial maps $q: \eta K \rightarrow \eta c K$ and $q: \eta L \rightarrow \eta c L$ where $q(\eta A)=\eta c A$ for $A \in K$ or $L$. Note that $q \circ f=c f \circ q$, and $q(D(A ; L))=$ $D(c A ; c L)$. Hence, $q: D(A ; f) \cong D(c A ; c f)$. Hence $D(c A ; c f) \searrow$ point. Since the collapse of $D(A ; f)$ is homogeneous in $K$ and since $d(q(x) ; c K)=$ $\operatorname{dim}(x ; K)+1$ for all $x$, the corresponding collapse of $D(c A ; c f)$ is homogeneous in $c K$.

Transverse cellularity follows from Proposition 3.

An application is the following uniqueness theory for the homeomorphism associated to a transverse cellular map. 
We say that $f_{0}, f_{1}: X \rightarrow Y$ are quasi-concordant if there exists $F$ : $(X \times I ; X \times 0, X \times 1) \rightarrow(Y \times I ; Y \times 0, Y \times 1)$ transverse cellular with $F^{-1}(X \times i)$ $=X \times i$ and $F_{i}=f_{i}, i=0,1$. If $F$ is also level-preserving, we will call $F$ a quasi-isotopy. The importance of this equivalence relation is the following:

Lemma 14. Let $f: K \rightarrow L$ be a transverse cellular simplicial map and let $\hat{f}: K \rightarrow L$ be a p.l. homeomorphism carried by $\gamma_{f}$. Then $f$ and $\hat{f}$ are quasiisotopic.

Proof. Consider the cone complexes $\{D(A ; f) \times i, D(A ; f) \times I: A \in L, i=$ $0,1\}$ and $\{D(A ; L) \times i, D(A ; L) \times I: A \in L, i=0,1\}$ on $K \times I$ and $L \times I$ respectively and let $\gamma$ be the obvious isomorphism of cone complexes. Extend $f \times 0$ and and $\hat{f} \times 1$ to a map of $K \times I \rightarrow L \times I$, carried by $\gamma$, by proceeding up the cells coning inductively with cone points at the $1 / 2$ level using the Alexander trick. $F$ is transverse cellular on $D(A ; f) \times 1$ 's and is so on the $D(A ; f) \times 0$ 's by the remarks following Proposition 10. On the remaining cells, the $D(A ; f) \times I$ 's, $F$ is transverse cellular by induction, i.e. $F$ is transverse cellular on $\dot{D}(A ; f) \times I \cup$ $D(A ; f) \times I$ by induction and Proposition 11 , so it is on $D(A ; f) \times I$ by Lemma 13 . Another application of Proposition 11 proves that $F$ is transverse cellular on $X \times I$.

Note that $F$ as constructed is level-preserving, i.e. $F$ is a quasi-isotopy.

Proposition 15. (1) Two bomeomorphisms are quasi-concordant iff they are concordant.

(2) Two bomeomorphisms associated to a transverse cellular map are concordant.

(3) A bomeomorphism is quasi-concordant to a transverse cellular map iff it is concordant to an associated bomeomorphism.

(4) Transverse cellular maps are quasi-concordant iff associated bomeomorphisms are concordant.

Proof. Proposition 2.

For a strengthening of these results in the compact case to their analogues for quasi-isotopies see the last section. Also we will show that our definition of quasi-isotopy is equivalent to the more natural definition: a level-preserving map $F$ with $F_{t}$ transverse cellular for all $t$ (Corollary V. 10).

V. Regular extensions. We construct for the p. 1. category the proper way of attaching one polyhedron by a map of a subpolyhedron to another one. Topologically if $f: \quad X_{0} \rightarrow Y$ with $X_{0} \subset X$ then we can think of $X \cup, Y$ as $X / f \cup Y$ where $X / f$ is obtained by "crushing" $X_{0}$ by the equivalence relation $x \equiv x^{\prime}$ means $f(x)=f\left(x^{\prime}\right)$, and where the union, $X / f \cup Y$ has intersection $X_{0} / f=$ $f\left(X_{0}\right)$. We generalize this to the simplicial category following Cohen. 
We call a subcomplex $K_{0}$ of $K$ well situated if $A \in K$ with $A \cap K_{0}=\varnothing$ implies $L k(A ; K) \cap K_{0}$ is empty or a simplex. For example, if $K_{0}$ is a full subcomplex of $K$, then $K_{0}$ is well situated in $\eta_{K_{0}} K$ where $\eta_{K_{0}}$ is a derived modulo $K_{0}$.

Suppose that $K_{0}$ is well situated in $K=N\left(K_{0} ; K\right) \cup P,\left(P \cap N\left(K_{0} ; K\right)=\right.$ $\left.\dot{N}\left(K_{0} ; K\right)\right)$ and that $f: K_{0} \rightarrow L_{0}$ is a simplicial mapping, then the stellar extension $F: K \rightarrow L$ is defined to be the unique, simplicial map of $K \rightarrow$ $\left(L_{0} * \dot{N}\left(K_{0} ; K\right)\right) \cup P$ which is the identity on $P$ and $f$ on $K_{0}$, where $L$ is defined to be the image of this map $\bigcup L_{0}$. Note that $L$ is locally finite and $F$ is proper.

Example. $C\left(d_{f}\right): C_{\mathrm{id}} \rightarrow C_{f}$ is a stellar extension of $f: K \rightarrow L$ ( $K$ regarded as range of $\mathrm{id}_{K}$ ).

The key property of stellar extensions, which relates them to transverse cellular maps is given by the following results of Cohen which calculates the preimages of points under a stellar extension $\left[C_{1}, 3.3\right]$.

Proposition 1. Let $K_{0}$ be a well-situated subcomplex of $K, f: K_{0} \rightarrow L_{0}$ a simplicial map and $F: K \rightarrow L$ the stellar extension of $f$. Then:

(1) for $y \in L_{0}, F^{-1}(y)=f^{-1}(y) ; y \in P, F^{-1}(y)=y ; y \in N\left(L_{0} ; L\right)-$ $\left(L_{0} \cup P\right), F^{-1}(y)$ is a convex cell.

(2) If $K=\eta_{K_{0}} \bar{K}$ then for $y \in N\left(L_{0} ; L\right)-\left(L_{0} \cup P\right), F^{-1}(y)$ is contained in the interior of a simplex of $\vec{K}$ and so $F:\left|K-K_{0}\right| \rightarrow\left|L-L_{0}\right|$ is transverse cellular.

Proof. (1) is $\left[C_{1}, 3.3\right]$ and the first part of (2) is an easy extension of that proof. The transverse cellularity follows from Corollary IV.4.

Just as in the case of regular neighborhood theory where we must sharpen the notion of stellar neighborhood to obtain p. 1. invariance so also in the case of stellar extension of a map.

If $K_{0}$ is a full subcomplex of $K, f: K_{0} \rightarrow L_{0}$ an onto simplicial map and $\eta_{K_{0}} K$ a derived $\bmod K_{0}$, then the stellar extension, . $F: \eta_{K_{0}} K \rightarrow L$, associated to $f$, is called a derived stellar extension, or a derived extension of $f$. Using derived extensions we will be able to develop the corresponding $\mathrm{p}$. 1 . theory.

Note that if $K=\Delta^{2}, K_{0}=\Delta^{1}$ and $L_{0}=\Delta^{0}$ then the image of $K$ under the stellar extension of $f: K_{0} \rightarrow L_{0}$ is a one simplex but the image after going to the derived is a two ball.

The theorem motivates our p. l. definition.

Definition. Let $X_{0}$ be a subpolyhedron of $X$ and $f: X_{0} \rightarrow Y_{0}$ a p. 1. map. A regular extension of $f$ is a map $F: X \rightarrow Y$ and an embedding $i: Y_{0} \rightarrow Y$ satisfying 
(1) $F \mid X_{0}=i \circ f$ and $F^{-1}\left(Y_{0}\right)=X_{0}$.

(2) $F: X-X_{0} \rightarrow Y-Y_{0}$ is transverse cellular.

We have seen that any derived stellar extension of a triangulation of $f$ gives a regular extension. This is "essentially" all there are.

Let $F_{1}: K \rightarrow L_{1}$ be a simplicial map with a full subcomplex $L_{0}$ of $L_{1}$ and $K_{0}=F_{1}^{-1}\left(L_{0}\right)$ (necessarily a full subcomplex of $K$ ). Choose deriveds $\bmod K_{0}$ and $L_{0}$ such that $F_{1}: \tau_{K_{0}} K \rightarrow \tau_{L_{0}} L_{1}$ is simplicial, then we can factor $F_{1}$, simplically, through the derived stellar extension $F: \tau_{K_{0}} K \rightarrow L$ of $F_{1} \mid K_{0}$ : $K_{0} \rightarrow L_{0}$. That is, there is a unique $G$, simplicial and making the following diagram commute:

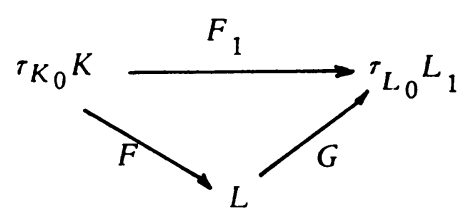

We use a special case of the following situation:

Lemma 2. Let $X \rightarrow{ }^{F_{1}} Y_{1} \leftarrow{ }^{i_{1}} Y_{0}$ and $X \rightarrow{ }^{F} Y \leftarrow{ }^{i} Y_{0}$ are regular extensions of $f: X_{0} \rightarrow Y_{0}$ and $G: Y \rightarrow Y_{1}$ such that $G^{-1} i_{1} Y_{0}=i Y_{0}$ and the following commutes:

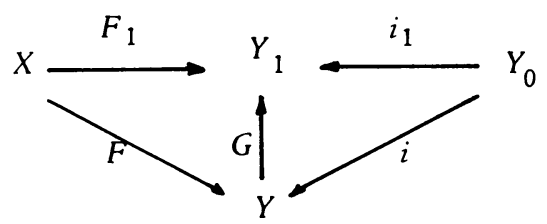

Then $G$ is transverse cellular.

Proof. Identify $Y_{0}$ with its images under $i_{,} i_{1}$ and triangulate to obtain the simplicial diagram

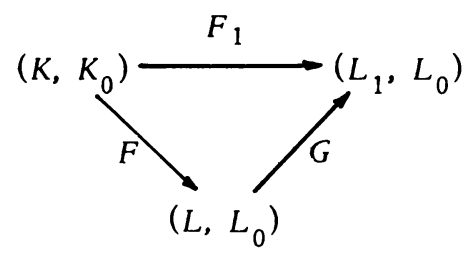

Let $A \in L_{1}$, then since $F_{1}=G F$, the definition of dual cells gives the commutative diagram:

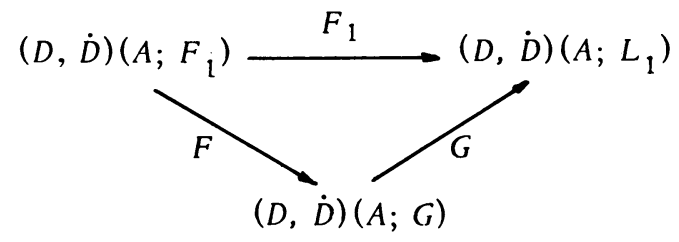

(1) $A \in L_{0}$, then since $G \mid L_{0}=\mathrm{id}_{L_{0}}$ and $G^{-1}\left(L_{0}\right)=L_{0},(D, \dot{D})(A ; G)=$ $(D, \dot{D})(A ; L)$ and is consequently a cone pair. For later reference note that in 
this case it then follows that $(D, \dot{D})\left(A ; F_{1}\right)=(D, \dot{D})(A ; F)$.

(2) $A \in L_{1}-L_{0}$, then by Proposition IV.10 $(D, \dot{D})\left(A ; F_{1}\right)$ is a cone pair.

But $D(A ; G)$ decomposes to the cone-complex $\{D(B ; L): A \leq G B\}$ and by applying $F^{-1}$, we decompose $D\left(A ; F_{1}\right)$ by $\{D(B ; F): A \leq G B\}$, which is also a cone complex by Proposition IV.10 applied to $F$. (Compare this with the proof of Proposition IV.12.) Realizing the obvious isomorphism gives a homeomorphism of pairs $(D, \dot{D})\left(A ; F_{1}\right) \cong(D, \dot{D})(A ; G)$. Since the former is a cone pair, the latter is.

Corollary 3. Let $f: X \rightarrow Y$ and $g: Y \rightarrow Z$ with $f$ and $g$ transverse cellular, then $g$ is transverse cellular.

Proof. $f$ and $g f$ are regular extensions of the "empty map" and Proposition 2 applies with $Y_{0}=0$.

Combining Corollary 3 and Corollary IV.12 we see that if $f$ and $g$ are transverse cellular then $g f$ is and if $f$ and $g f$ are transverse cellular then $g$ is. However, $g$ and $g f$ may be transverse cellular and $f$ not so. For let $Y$ be a manifold (with $\partial Y=0$ ) and $B$ be a codimension 0 ball in $Y$. If $g$ is a regular extension of $B \rightarrow$ point then $g$ has collapsible point-inverses and is thus transverse cellular. Now if $f: Y \rightarrow Y$ with $f^{-1}(B)=B$ and $f \mid Y-B=$ identity then $g f=g$ and is thus transverse cellular. But since $f$ can do anything inside the ball it clearly need not be transverse cellular itself.

We now apply Lemma 2 to obtain the "uniqueness theorem" for regular extensions.

Proposition 4. Let $X_{0}$ be a subpolybedron of $X$ and $f: X_{0} \rightarrow Y_{0}$ be a p. l. map.

(1) A p. l. map $F_{1}: X \rightarrow Y_{1}$ and an embedding $i: Y_{0} \rightarrow Y_{1}$ satisfying $F_{1} \mid X_{0}=i \circ f$ and $F^{-1}\left(Y_{0}\right)=X_{0}$ is a regular extension of $f$ iff there exists a derived stellar extension of a triangulation of $f, F: X \rightarrow Y$, and a transverse cellular map $G: Y \rightarrow Y_{1}$ with $G^{-1}\left(i\left(Y_{0}\right)\right)=Y_{0}$ and $G F=F_{1}$.

(2) Let $F_{1}: X \rightarrow Y_{1}, i_{1}: Y_{0} \rightarrow Y_{1}$ and $F_{2}: X \rightarrow Y_{1}, i_{2}: Y_{0} \rightarrow Y_{2}$ be regular extensions of $f$. Then there exists a regular extension $F: X \rightarrow Y$, $i: Y_{0} \rightarrow Y$ and transverse cellular maps $G_{\alpha}: Y \rightarrow Y_{\alpha}$ with $G_{\alpha}^{-1}\left(i_{\alpha}\left(Y_{0}\right)\right)=$ $i\left(Y_{0}\right)$ for $\alpha=1,2$ and making the following diagram commute:

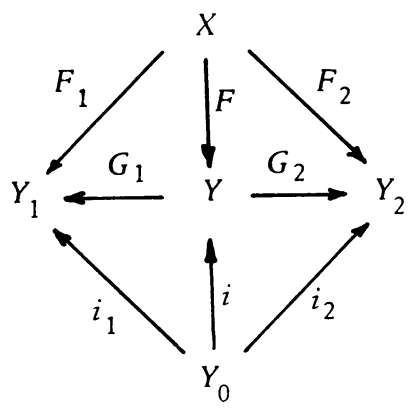


Proof. (1) follows from Lemma 2 one way and Corollary IV.12, the other.

(2) follows directly from Lemma 2 , just triangulate so that $F_{1}$ and $F_{2}$ are simultane ously simplicial.

Remark. For a rather different "uniqueness theorem" for regular extensions see Proposition VI.9.

One might suspect that we have allowed too much with transverse cellular maps and that if we restricted our definition to triangulations of derived stellar extensions we might be able to get a uniqueness theorem relating two extensions by conjugation with a homeomorphism. This is not usually possible as the following example shows.

Let $\left(K, K_{0}\right)=\left(\Delta^{2}, \Delta^{1}\right), L_{0}=\Delta^{0}$ and $K^{*}$ equals stellar subdivision of $K$ at $\Delta^{1}$. Then if $F: \eta_{\Delta^{1}} K \rightarrow L$ and $F^{*}: \eta_{\Delta^{1}} K^{*} \rightarrow L^{*}$ are the derived stellar extensions then the singularity sets are not homeomorphic. $S(F)$ is a 2 -simplex and $S\left(F^{*}\right)$ is two 2-simplices joined at a vertex.

Of occasional usefulness is the following feeble analogue of Cohen's powerful result for regular neighborhoods $\left[C_{2}, 6.1\right]$.

Lemma 5 (Stellar Extension Lemma). Let $K_{0}$ be well situated in $K$ and $f$ : $K_{0} \rightarrow L_{0}$ a simplicial map with $F: K \rightarrow L$ the stellar extension of $f$. Any simplex $A \in N\left(K_{0} ; K\right)-\left(K_{0} \cup \dot{N}\right)$ can be written $\underset{\sim}{A}=B C$ where $B=A \cap K_{0}$. If for any such $A$ all faces $\widetilde{B} C$, where $\widetilde{B}<B$ and $f(\widetilde{B})=f(B)$, are nice faces (see [A, II 12]), then $F:|K| \rightarrow|L|$ is a regular extension of $f$.

Proof. Referring to $\left[C_{1}, 3.3\right]$ as in the proof of part (2) of Proposition 1, we see that this is precisely the condition needed to insure constant intrinsic dimension of all convex cell $F^{-1}(y)$ for $y \in N\left(L_{0} ; L\right)-\left(L_{0} \cup \dot{N}\right)$.

Regular extensions provide a tool for constructing transverse cellular maps.

Proposition 6. Let $X_{0}$ be a subpolybedron of $X$ and $f: X_{0} \rightarrow Y_{0}$ a p.l. map. Let $K \rightarrow{ }^{F} L \leftarrow^{i} L_{0}$ be the triangulation of a regular extension of f, i.e. $|K|=X,\left|L_{0}\right|=Y$ and $\left|K_{0}\right|=X_{0}$. Then the following are equivalent.

(1) For all $A \in i\left(L_{0}\right), D(A ; F) \searrow$ point bomogeneously in $X$.

(2) $F: K \rightarrow L$ is transverse cellular.

(3) For every regular extension of $f_{0} X \rightarrow{ }^{F_{1}} Y_{1} \leftarrow^{i}{ }^{1} Y_{0}, F_{1}$ is transverse cellular.

Proof. By Proposition IV. 10 (1) is equivalent to (2). Clearly, (3) implies (2).

$(2) \Rightarrow(3)$. By Proposition 4.(2) we can obtain a regular extension $X \rightarrow{ }^{F_{2}} Y_{2} \leftarrow{ }^{i} 2 Y_{0}$ "between" $F$ and $F_{1}$. We will show that $F_{2}$ is transverse cellular, whence $F_{1}$ will be by Corollary IV.12.

Triangulate so that $F_{2}, F$ and the connecting transverse cellular map $G$ : $Y_{2} \rightarrow|L|$ are simplicial. For $\sigma$ a simplex of $\left|L_{0}\right|$, we know that $D(i \sigma ; F)$ is a 
cone on its boundary and we must show this for $D\left(i_{2} \sigma ; F_{2}\right)$. But just as in the proof of Lemma 2, part (1) $(D, \dot{D})\left(i_{2} \sigma, F_{2}\right)=(D, \dot{D})(i \sigma, F)$. So $F_{2}$ satisfies condition (1) and as (1) $\Rightarrow(2), F_{2}$ is transverse cellular.

Proposition 7. Let $K_{0}$ be a full subcomplex of $K$, and $f: K_{0} \rightarrow L_{0} a$ simplicial map. If for all $A \in L_{0}, D(A ; f) \searrow$ point bomogeneously in $K$, then any regular extension of $f$ is transverse cellular.

Proof. By Proposition 6 we need only show the derived stellar extension $F$ : $\tau_{K_{0}} K \rightarrow L$ satisfies $D(A ; F) \searrow$ point homogeneously in $K$ for $A \in L_{0}$. But $D(A ; F) \searrow D(A ; f)$ homogeneously in $K$ because $F^{-1} \eta A=f^{-1} \eta A$ and the relative version of Proposition III.2, mentioned in the remark following it, imply that $D(A ;(F, f))$ is a regular neighborhood pair of $f^{-1} \eta A$ in $\dot{D}(\bar{A} ;(F, f))$. Collapsing follows $\left[C_{2}, 7.10 \mathrm{a}\right]$ and [A, III. 11].

Beware: Homogeneity of a collapse in $K$ and in $K_{0}$ are independent conditions. In particular, $f: K_{0} \rightarrow L_{0}$ transverse cellular need not imply $F$ transverse cellular.

We conclude this section with the converse of IV.12 promised earlier.

Proposition 8. Let $f: X \rightarrow Y$ be a transverse cellular map with $U \subset Y$ subpolybedron, $V=f^{-1}(U) \subset X$ with Frontier $_{Y} U \equiv \dot{U}$ and Frontier $_{X} V \equiv \dot{V}$. Assume the following conditions bold.

(a) $\dot{V}=f^{-1}(\dot{U})$.

(b) Let $C(U ; Y)=Y$ - Int $U=(Y-U) \cup \dot{U}$ similarly $C(V ; X)$, then

$$
U \supset \dot{U} \subset C(U ; Y), \quad V \supset \dot{V} \subset C(V ; X)
$$

are assumed to be locally collared inclusions.

Then, $f:(X ; V, C(V ; X), \dot{V}) \rightarrow(Y ; U, C(U ; Y), \dot{U})$ is transverse cellular.

Proof. Triangulate to obtain $f: K \rightarrow L$ simplicial with $L_{0}=U$ a subcomplex and hence $\dot{L}_{0}=\dot{U}$ a complex. Let $K_{0}=V=f^{-1}\left(L_{0}\right)$ and $\dot{K}_{0}=\dot{V}=$ $f^{-1}\left(\dot{L}_{0}\right)$.

If $A \in L-\dot{L}_{0}$ then

$$
D(A ; f)= \begin{cases}D\left(A ; f \mid K_{0}\right), & A \in L_{0}, \\ D\left(A ; f \mid C\left(K_{0} ; K\right)\right), & A \notin L_{0} .\end{cases}
$$

Hence, since $f$ is transverse cellular each of these two collapses as required. We need only worry about simplices of $\dot{L}_{0}$. In fact, because $f^{-1}\left(\dot{L}_{0}\right)=\dot{K}_{0}$ we know $D(A ; f) \searrow D\left(A ; f \mid \dot{K}_{0}\right)$ homogeneously in $K$ and hence $D\left(A ; f \mid K_{0}\right) \searrow$ $D\left(A ; f \mid \dot{K}_{0}\right)$ and $D\left(A ; f \mid C\left(K_{0} ; K\right)\right) \searrow D\left(A ; f \mid \dot{K}_{0}\right)$ (by local collaring $D(A ; f)$ $D\left(A ; f \mid \dot{K}_{0}\right)$ has two components with these closures $)$. So it suffices to know $f \mid \dot{K}_{0}: \dot{K}_{0} \rightarrow \dot{L}_{0}$ is transverse cellular. 
Now we use the natural retractions: $T_{1}: N\left(\dot{K}_{0} ; \eta K_{0}\right) \rightarrow \eta \dot{K}_{0}$ and $T_{2}$ : $N\left(\dot{L}_{0} ; \eta L_{0}\right) \rightarrow \eta \dot{L}_{0}$. We have the commutative diagram:

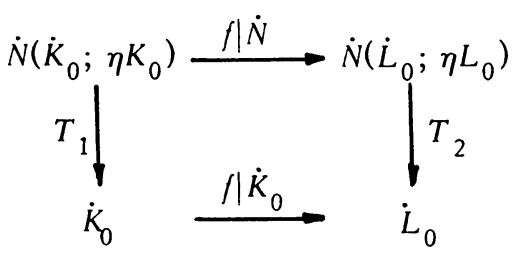

Now $f \mid \dot{N}$ is the union of maps $f \mid D(A ; f)$ for $A \in N\left(\dot{L}_{0} ; L_{0}\right)-\left(\dot{N} \cup \dot{L}_{0}\right)$. Since $f$ is transverse cellular on $(D(A ; f), \dot{D}(A ; f))$ it is transverse cellular on $\dot{N}\left(\dot{K}_{0} ; \eta K_{0}\right)$ by the remarks to Proposition IV.11.

On the other hand the maps $T_{1}$ and $T_{2}$ are transverse cellular, by Corollary VI.5 of the next section, and so, by Corollary $3 f \mid \dot{K}_{0}$ is also transverse cellular.

Remarks. (1) Note that in this proof if we only assumed $\dot{U} \subset U$ and $\dot{V} \subset V$ collared then we would obtain $f:(V, \dot{V}) \rightarrow(U, \dot{U})$ transverse cellular. We only use local collaring on the outside to insure that the homogeneous (in $K_{0}$ ) collapse of $D\left(A ; f \mid \dot{K}_{0}\right) \searrow$ point is also homogeneous in $\left(C\left(K_{0} ; K\right), \dot{K}_{0}\right)$.

(2) If $\mathrm{f}: K \rightarrow L$ is a transverse cellular simplicial map with chosen derived and $L_{0}$ is a full subcomplex of $L, f^{-1} L_{0}=K_{0}$, then the theorem applies with $U=N\left(L_{0} ; \eta L\right)$ and $V=N\left(K_{0} ; \eta K\right)$.

(3) Iterating the result gives a theorem in the following situation. Let $f$ : $X \rightarrow Y$ be a transverse cellular map and define sequences: $Y=\dot{Y}_{-1} \supset Y_{0} \supset \dot{Y}_{0} \supset$ $Y_{1} \supset \dot{Y}_{1} \supset \ldots \supset Y_{n} \supset \dot{Y}_{n}$ and $X_{i}=f^{-1}\left(Y_{i}\right), \dot{X}_{i}=f^{-1}\left(\dot{Y}_{i}\right)$ where $Y_{i}$ is a regular neighborhood with boundary $\dot{Y}_{i}$ in $\dot{Y}_{i-1}$ for $i=0, \ldots, n$ and similarly for $X_{i}$ (respectively, $Y_{i}-\dot{Y}_{i}$ is open in $\dot{Y}_{i-1}$ with $\dot{Y}_{i} \subset Y_{i}$ collared for $i=0$, $\ldots, n$ and similarly for $X_{i}$ ). By Proposition 8 ,

$$
f:\left(\dot{X}_{i-1} ; X_{i}, \overline{\dot{X}_{i-1}-X_{i}}, \dot{X}_{i}\right) \rightarrow\left(\dot{Y}_{i-1} ; Y_{i}, \overline{\dot{Y}}_{i-1}-Y_{i}, \dot{Y}_{i}\right)
$$

is transverse cellular for $i=0, \ldots, n$ (respectively, by Remark (1) $f$ : $\left(X_{i}, \dot{X}_{i}\right) \rightarrow\left(Y_{i}, \dot{Y}_{i}\right)$ is transverse cellular for $\left.i=0, \ldots, n\right)$. From this follows the promised converse:

Proposition 9. Let $f: X \rightarrow Y$ be a transverse cellular map and assume $\gamma: \Theta \rightarrow \supseteqq$ is an is omorphism of general decompositions carrying f, i.e. $f(\sigma)=$ $\gamma(\sigma)$, and $f^{-1}(\partial y \sigma)=\partial \sigma$. Then $f \mid \sigma:(\sigma, \partial \sigma) \rightarrow(\gamma \sigma, \partial \gamma \sigma)$ is transverse cellular.

This allows us also to verify the satisfying definition of quasi-isotopy mentioned after Proposition IV.15.

Corollary 10. Let $F: X \times I \rightarrow Y \times I$ be a map with $F^{-1}(Y \times i)=X \times i$ for $i=0,1$. Then the following are equivalent: 
(1) F is transverse cellular.

(2) $F:(X \times I ; X \times 0, X \times 1) \rightarrow(Y \times I ; Y \times 0, Y \times 1)$ is transverse cellular.

If $F$ is level-preserving and $X$ and $Y$ are compact these are equivalent to:

(3) $F_{t}: X \rightarrow Y$ is transverse cellular for all $t \in I$.

Proof. (1) $\Rightarrow$ (2) by Remark (1) after Proposition 8 letting $(U, \dot{U})=$ $(Y \times I, Y \times I)$. Now assume $F$ level-preserving.

$(1) \Rightarrow$ (3). Let $J$ be any subinterval of $I$, then $F:(X \times J, X \times j) \rightarrow$ $(Y \times J, Y \times j)$ is transverse cellular by Remark (1) af ter Proposition 8. A fortiori, $F_{t}: X \rightarrow Y$ is transverse cellular for each $t$ in $l$.

(3) $\Rightarrow(1)$. Triangulate so that $X \times I \rightarrow^{F} Y \times I \rightarrow^{\pi} I$ is simplicial (this needs compactness, else $\pi$ is not proper). Choose deriveds preserving the diagram. If $A \in Y \times I$ with $\pi A=t$ a zero simplex, then $D(A ; f) \searrow D(A ; F \mid X \times t)$ homogeneously in $X \times I$, as $F^{-1} Y \times t=X \times t$. Since $F_{t}$ is transverse cellular, $D(A ; F \mid X \times t) \searrow$ point homogeneously in $X \times t$ and hence in $X \times I$. If $\pi A=J$ a one simplex, then since $F_{\eta J}$ is transverse cellular and $D(A ; F) \subset X \times \eta J, F$ : $D(A ; F) \rightarrow D(A ; L \times I)$ is transverse cellular by Proposition 9, using $\{D(B ; F)$ : $\pi B=J\}$ and $\{D(B ; L \times I): \pi B=J\}$ as general complexes on $X \times \eta J$ and $Y \times \eta J$ respectively. Since $D(A ; L \times I)$ is a cone on $\dot{D}, D(A ; F)$ is.

VI. Classification of mapping cylinders. We begin by applying the result on regular extensions to mapping cylinders. First, the relation between regular extensions and mapping cylinders.

Proposition 1. Let $f: K \rightarrow L$ and $g: L \rightarrow M$ be simplicial maps with chosen deriveds, with $g$ onto. Call $\bar{g}: f \rightarrow g$ of the morphism defined as the pair $\left(\mathrm{id}_{K}, g\right.$ ). The map $C(\bar{g}): C_{f} \rightarrow C_{g \circ f}$ is a regular extension of $g: L \rightarrow M$.

Proof. We apply the criterion of Lemma V.5 and we must show for $A \eta C_{0} \cdots \eta C_{k} \in C_{f}$ and $\tilde{A}<A$ such that $g(A)=g(\tilde{A})$, that $\tilde{A} \eta C_{0} \cdots \eta C_{k}$ is a nice face of $A \eta C_{0} \cdots \eta C_{k}$ in $C_{f}$ (since this is for any $g$, the condition $g(\tilde{A})=$ $g(A)$ gives no information, and we do not use it). But

$$
\begin{aligned}
& L k\left(A \eta C_{0} \cdots \eta C_{k} ; C_{f}\right)=L k\left(A \eta C_{0} ; C_{f \mid C_{0}}\right) * L k\left(\eta C_{0} \cdots \eta C_{k} ; D\left(C_{0} ; K\right)\right), \\
& L k\left(\widetilde{A}_{\eta} C_{0} \cdots \eta C_{k} ; C_{f}\right)=L k\left(\widetilde{A}_{\eta} C_{0} ; C_{f \mid C_{0}}\right) * L k\left(\eta C_{0} \cdots \eta C_{k} ; D\left(C_{0} ; K\right)\right) .
\end{aligned}
$$

The latter join factor is held in common. So to prove the result, we need only show that $\tilde{A}_{\eta} C_{0}$ is a nice face of $A \eta C_{0}$ in $C_{f} \mid C_{0}$. But $C_{f} \mid C_{0}$ is a $\operatorname{dim} C_{0}+1$ ball with boundary $C_{0} \cup f C_{0} \cup C_{f \mid \partial C_{0}}$ so both $\tilde{A \eta} C_{0}$ and $A \eta C_{0}$ are interior simplices.

Note here-as I failed to in my original manuscript-that $g$ can be transverse cellular without $C(\bar{g})$ being transverse cellular. I was not wary of the misuse of 
Proposition V.7, mentioned after its proof. However, a weaker result is true and useful.

Corollary 2. Let $g: K \rightarrow L$ be a transverse cellular simplicial map. Then $\eta C\left(d_{g}\right) \circ j_{K}^{-1}: \eta K \times I \rightarrow \eta C_{g}$ is transverse cellular.

Proof. $j_{K}$ is an isomorphism and $C\left(d_{g}\right)$ is transverse cellular, by the above Propositions V.6 and V.7. Note that for $A \in L, D(A ; g)$ collapses homogeneously in $K \times 1$ by transverse cellularity and hence in $C\left(\right.$ id $\left._{K}\right)$ because $K \times 1$ is collared in $C\left(\right.$ id $\left._{K}\right)$.

There is a corresponding morphism $\bar{f}: g \circ f \rightarrow g$ given by the pair of maps $\left(f, \mathrm{id}_{M}\right)$ but the corresponding map $C(\bar{f}): C_{g \circ f} \rightarrow C_{g}$ is in general not a regular extension of $f$ if $f$ is not transverse cellular. For in particular if $C(\bar{f})$ were a regular extension then it would be transverse celluiar on a complement of a regular neighborhood of $\eta K$ to the complement of a regular neighborhood of $\eta L$. By restricting to the boundaries we would obtain a transverse cellular map of $K$ onto $L$ and hence that $K$ is homeomorphic to $L$. Oddly, the analogue of Corollary 2 can be strengthened.

Proposition 3. Let $f: K \rightarrow L$ and $g: L \rightarrow M$ be simplicial maps with chosen derived, with $f$ transverse cellular. Then $C(\bar{f}): C_{g \circ f} \rightarrow C_{g}$ is transverse cellular.

Proof. $C(\bar{f})$ preserves the decomposition maps:

$$
D(A ; g \circ f) \rightarrow D(A ; g), \quad Q(A ; g \circ f) \rightarrow Q(A ; g), \text { for } A \in M
$$

and $C(\bar{f})$ on $Q(A ; g \circ f)$ is the cone on $C(\bar{f}) \mid \dot{Q}(A ; g \circ f) \cup D(A ; g \circ f)$.

Since $D(A ; g)$ is a union of $D(B ; L)$ 's, $D(A ; g \circ f)$ is a union of $D(B ; f)$ 's. Now $f$ is transverse cellular mapping $D(B ; f) \rightarrow D(B ; L)$ for $B \in L$ by Proposition IV.10 and remarks thereafter. Hence, by Proposition IV.11, $f: D(A ; g \circ f)$ $\rightarrow D(A ; g)$ is transverse cellular.

Hence, $C(\bar{f})$ is transverse cellular on each $D(A ; g \circ f)$ and hence by Proposition IV.11 and Lemma IV.13 applied to an induction up the dimension of the $Q(A ; g \circ f)$ 's, $C(\bar{f})$ is transverse cellular on each of these pieces. The map is then transverse cellular on the whole thing by yet another application of Proposition IV.11.

We now prove the mapping cylinder regular neighborhood relation that we mentioned earlier. It is essentially $\left[C_{1}, 9.7\right]$, but his retraction is a little different and for later work we must have the result for the natural retraction of a simplicial neighborhood.

Proposition 4. Let $K_{0}$ be a full subcomplex of $K$ and let $T: N\left(K_{0} ; \eta K\right) \rightarrow$ $\eta K_{0}$ be the simplicial retraction defined by $T(\eta A)=\eta\left(A \cap K_{0}\right)$ for $A \epsilon$ $N\left(K_{0} ; K\right)$. 


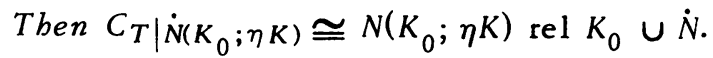

Proof. Define $A^{\eta}$, for $A \in K$, to be the subcomplex of $\eta K$ obtained by subdividing the simplex $A$. Let $U=N\left(K_{0} ; K\right)-\left(K_{0} \cup \dot{N}\left(K_{0} ; K\right)\right)$.

By $\left[C_{2}, 2.16\right]$ the following is a cell-complex on $N\left(K_{0} ; \eta K\right)$ :

$$
\left\{N\left(K_{0} \cap A ; A^{\eta}\right): A \in U\right\} \cup\left\{\dot{N}\left(K_{0} \cap A ; A^{\eta}\right): A \in U\right\} \cup K_{0},
$$

and we claim that the following is a cell-complex on $C_{T \mid \dot{N}}$.

$$
\left\{C_{T \mid \dot{N}\left(K_{0} \cap A ; A \eta\right)}: A \in U\right\} \cup\left\{\dot{N}\left(K_{0} \cap A ; A^{\eta}\right): A \in U\right\} \cup K_{0} .
$$

The result will then follow that by realizing the obvious cell-complex isomorphism.

To show that we have a cell-complex on $C_{T \mid \dot{N}}$ we must show that $C_{T \mid \dot{N}\left(K_{0} \cap A ; A \eta\right)}$ is a $\operatorname{dim} A$ ball with boundary equal to $C_{T \mid \dot{N}\left(K_{0} \cap A ; \partial A \eta\right)} U$ $\left(K_{0} \cap A\right) \cup \dot{N}\left(K_{0} \cap A ; A^{\eta}\right)$. For this we show that $T: \dot{N}\left(K_{0} \cap A ; A^{\eta}\right) \rightarrow K_{0} \cap$ $A$ has collapsible point-inverses. This and Proposition 1 allow us to apply Corollary IV.6 to the map

$$
\dot{N}\left(K_{0} \cap A ; A^{\eta}\right) \times I \stackrel{C\left(d_{T}\right)}{\longrightarrow} C_{T \mid \dot{N}\left(K_{0} \cap A ; A^{\eta}\right)}
$$

as in the proof of Proposition IV.9 (closed).

We are reduced to looking at the map $T: \dot{N}\left(K_{0} \cap A ; A^{\eta}\right) \rightarrow A \cap K_{0}$.

To analyze it, let $A=B D$ with $B=A \cap K_{0}$. Define a simplicial isomorphism so that the following diagram commutes:

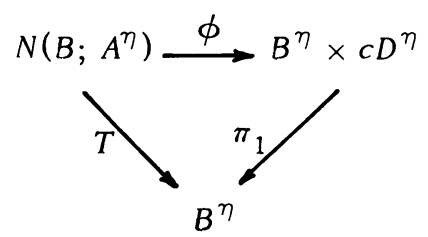

Let

$$
\phi\left(\eta\left(B^{\prime} D^{\prime}\right)\right)= \begin{cases}\left(\eta\left(B^{\prime}\right), \eta\left(D^{\prime}\right)\right), & \text { for } 0 \neq B^{\prime} \leq B \text { and } 0 \neq D^{\prime} \leq D, \\ \left(\eta\left(B^{\prime}\right), c\right), . & \text { for } 0=D^{\prime} .\end{cases}
$$

Note that $\phi\left(\dot{N}\left(B ; A^{\eta}\right)\right)=B^{\eta} \times D^{\eta}$.

So if $x \in A \cap K_{0},(T \mid \dot{N})^{-1}(x)$ is a ball because $\left(\pi_{1} \mid B^{\eta} \times D^{\eta}\right)^{-1}(x)=x \times$ $D^{\eta}$ is.

Corollary 5. Let $\left(K, K_{0}\right)$ be a triangulation of $(X \times J, X \times p)$ for $J$ a real interval with endpoint p. Assume $K_{0}$ is full. Then $T: \dot{N}\left(K_{0} ; \eta K\right) \rightarrow \eta K_{0}$ is a transverse cellular mapping.

Proof. By Corollary 4 the mapping cylinder is homeomorphic to the regular neighborhood which is a product. $T$ is transverse cellular by Proposition IV.10. 
Remark. Uniqueness of regular neighborhoods gives a homeomorphism $b$ : $X \times J \rightarrow X \times J$ rel $X \times p$, with $b\left(N\left(K_{0} ; \eta K\right)\right)=X \times J^{\prime} \quad\left(J^{\prime}=[p, q]\right.$ a subinterval of $J)$. Then $b \mid \dot{N}: \dot{N}\left(K_{0} ; \eta K\right) \rightarrow X \times q \rightarrow X$ is a homeomorphism and we claim that $b$ is quasi-concordant to $T \mid \dot{N}$. Consider the composed map:

$$
\dot{N}\left(K_{0} ; \eta K\right) \times I \stackrel{C\left(d_{T}\right)}{\longrightarrow} C_{T \mid \dot{N}} \cong N\left(K_{0} ; \eta K\right) \stackrel{b}{\longrightarrow} X \times J^{\prime} \cong X \times I ;
$$

where $C\left(d_{T}\right)$ is transverse cellular by Corollary 2 and the last map is induced by an isomorphism of $J^{\prime}$ with $I$ taking $p$ to 0 and $q$ to 1 . Then on 0 , this composed map is $T \mid \dot{N}$ and on 1 it is the above homeomorphism.

We now classify mapping cylinders. Consider p. 1. maps from $X$ to $Y$. Define an equivalence relation $\sim$ generated by $f_{0} \sim_{e} f_{1}$ meaning there exists a transverse cellular map $r: X \rightarrow X$ quasi-concordant to $\mathrm{id}_{X}$ and such that $f_{0}=$ $f_{1} r$.

Theorem 6. Let $f_{0}, f_{1}: X \rightarrow Y$ be p. l. maps. The following are equivalent.

(1) $f_{0} \sim f_{1}$.

(2) There exists a bomeomorphism b: $C_{f_{0}} \cong C_{f_{1}}$ rel $X \cup Y$.

Proof. (1) $\Rightarrow(2)$ We may assume $f_{0} \sim_{e} f_{1}$ since condition (2) is transitive and symmetric. If $f_{0}=f_{1} r$, triangulate so that $f_{1}$ and $r$ (and hence $f_{0}$ ) are simplicial, by Proposition 3, $C(\bar{r}): C_{f_{0}} \rightarrow C_{f_{1}}$ is transverse cellular. In fact it is a transverse cellular map of $\left(C_{f_{0}} ; X, Y\right) \rightarrow\left(C_{f_{1}} ; X, Y\right)$ which is $r$ on $X$ and id $_{Y}$ on $Y$. So by Proposition IV.2, we can change $C(\bar{r})$ rel $Y$ to obtain a homeomorphism $\left(C_{f_{0}}, X\right) \rightarrow{ }^{b} 0\left(C_{f_{1}}, X\right)$. The homeomorphism so obtained on $X$ is quasiconcordant to $r$ and hence to $\mathrm{id}_{X}$ and so is concordant to id $X$ by Proposition IV.15. Since $X$ is collared in $C_{f_{0}}$ and $C_{f_{1}}$, we can change $b_{0}$ by a concordance rel $Y$ to obtain $b: C_{f_{0}} \cong C_{f_{1}}$ rel $X \cup Y$.

$(2) \Rightarrow(1)$. Let $b: C_{f} \cong C_{g}$ be a homeomorphism rel $X \cup Y$, for $f, g: X \rightarrow$ $Y$. For this proof, we let $d$ equal $C\left(d_{f}\right) j_{X}^{-1}: X \times I \rightarrow C_{f}$, and $\bar{d}$ equal $C\left(d_{g}\right) j_{X}^{-1}$. Subdivide so that the following diagram is simplicial:

$$
X \times I \stackrel{d}{\longrightarrow} C_{f} \stackrel{b}{\longrightarrow} C_{g} \stackrel{\bar{d}}{\stackrel{1}{\longrightarrow}} X \times I
$$

and choose deriveds so that it remains so:

$$
\eta X \times I \stackrel{d}{\longrightarrow} \eta C_{f} \stackrel{b}{\longrightarrow} \bar{\eta} C_{g} \stackrel{\bar{d}}{\longleftarrow} \bar{\eta}(X \times I) .
$$

Consider the following commutative diagram where the vertical maps are the natural simplicial retractions of Proposition 4 and Corollary 5 (in particular, note that $T_{2}$ and $T_{3}$ are not $P_{f}$ and $P_{g}$ ): 


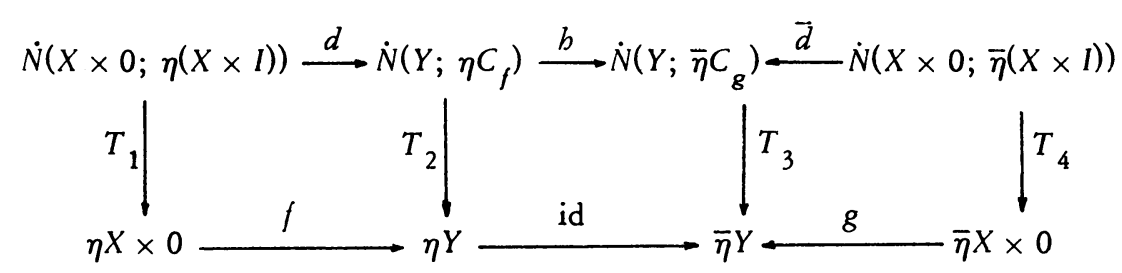

If we had allowed the domain space to vary in defining our equivalence relation we would now be done for then $f$ relates to $T_{2}$ relates to $T_{3}$ relates to $g$, as the other maps are transverse cellular. The remainder of the proof is obtaining the sharp form of the result that we stated above.

Essentially, we have cut the diagram of maps at the $\epsilon$-level and looked at the $[0, \epsilon]$ piece.

However, we have now to look at the part "above the cut", the complements of the regular neighborhoods $(C(A ; \eta B)=$ closure $(B-N(A ; \eta B)))$ :

$$
C(X \times 0 ; \eta(X \times I)) \stackrel{d}{\longrightarrow} C\left(Y ; \eta C_{f}\right) \stackrel{b}{\longrightarrow} C\left(Y ; \bar{\eta} C_{g}\right) \stackrel{\vec{d}}{\longrightarrow} C(X \times 0 ; \bar{\eta}(X \times I)) .
$$

Now by Corollary 2, $d: X \times I \rightarrow C_{f}$ is transverse cellular off $X \times 0$ and so Proposition V.8 and Remark (1), thereafter, implies that

$$
d:(C(X \times 0 ; \eta(X \times I)) ; \dot{N}(X \times 0 ; \eta(X \times I)), X \times 1) \rightarrow\left(C\left(Y ; \eta C_{f}\right) ; \dot{N}\left(Y ; \eta C_{f}\right), X\right)
$$

is transverse cellular, and similarly for $\bar{d}$. So we can change $d$ and $\bar{d}$ rel $X$ to homeomorphisms of triads $q$ and $\bar{q}$. We will also need homeomorphisms $k, \bar{k}$ : $X \times I \rightarrow X \times I$ rel $X \times I$ with $k(X \times[0,1 / 2]) \cong N(X \times 0 ; \eta(X \times I))$ and $\bar{k}(X \times[0,1 / 2]) \cong N(X \times 0 ; \bar{\eta}(X \times I))$.

This enlarges the $[0, \epsilon]$ diagram to the following:

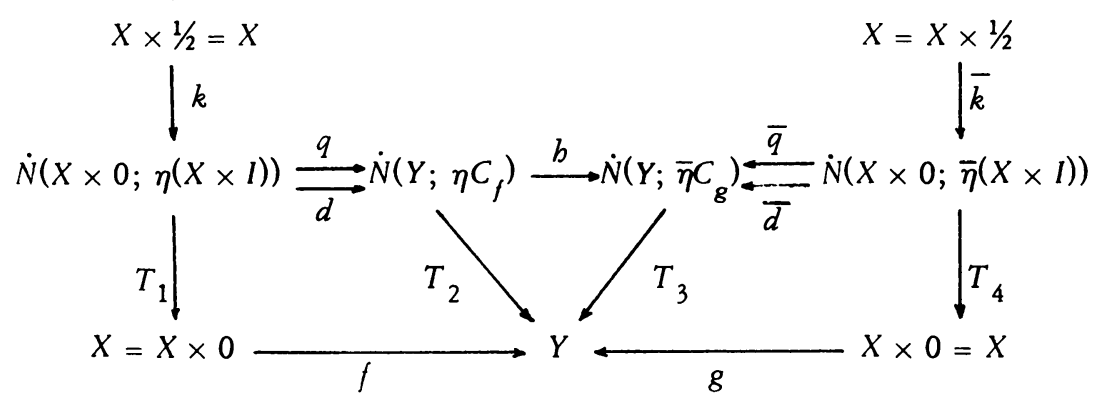

$f_{e} \sim f \circ T_{1} \circ k=T_{2} \circ d \circ k=T_{3} \circ b \circ d \circ k$ where $T_{1} \circ k$ is quasi-concordant to id $x$ by the remarks after Corollary 5. $T_{3} \circ b \circ q \circ k_{e} \sim T_{3} \circ b \circ q \circ k \circ$ $\left(k^{-1} q^{-1} d k\right)=T_{3} \circ b \circ d \circ k$ for since $q$ is quasi-concordant to $d, k^{-1} q^{-1} d k$ is quasi-concordant to id $X$.

Similarly, $g_{e} \sim T_{3} \circ \bar{d} \circ \bar{k} \sim_{e} T_{3} \circ \bar{q} \circ \bar{k}$.

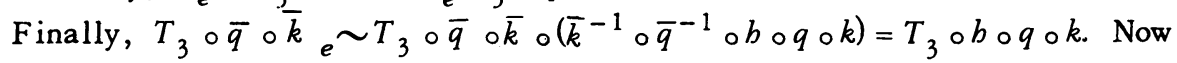


$\bar{k}^{-1} \circ \bar{q}^{-1} \circ b \circ q \circ k$ is concordant to the identity because it is the restriction to $X \times 1 / 2$ of a homeomorphism $X \times[1 / 2,1] \rightarrow \mathrm{X} \times[1 / 2,1]$ which is $\mathrm{id}_{X}$ on 1 .

This theorem may be of interest in examining thickenings of polyhedra. A thickening $(m \geq 6)$ of $X$ is a manifold $M^{m}$ together with an embedding $i: X \rightarrow$ Int $M$ which is a simple homotopy equivalence. More generally, an element $\mathfrak{J}^{m}(X)$ is a regular neighborhood pair $(M, X)$ where $M$ is an $m$ manifold. By Proposition 4, there exists $T: M \rightarrow X$, a retraction with $M \cong C_{T \mid \partial M}$ rel $\partial M \cup X$. The above theorem tells us how $T$ can vary, i.e. $(M, X)$ determines $T$ up to equivalence $\sim$. For any real usefulness, though, we would seem to need a theory of when an onto map $T: N \rightarrow X, N$ a manifold $(\partial=0)$ and $X$ a polyhedron, has $C_{T}$ a manifold (note that since $N \subset C_{T}$ is collared so $N \subset \partial C_{T}$ ).

Theorem 6 weakens to obtain other equivalence relation results. Let $f_{0}$ : $X_{0} \rightarrow Y_{0}$ and $f_{1}: X_{1} \rightarrow Y_{1}$.

(1) Define $f_{0} \approx_{e} f_{1}$ if $Y_{0}=Y_{1}$ and there exists $r: X_{0} \rightarrow X_{1}$ transverse cellular with $f_{0}=f_{1} \circ r$. Call the generated equivalence relation $\approx$.

(2) Define $f_{0} \approx_{e} f_{1}$ if there exists $r: X_{0} \rightarrow X_{1}$ transverse cellular and $b: Y_{1} \rightarrow Y_{0}$ a homeomorphism with $f_{0}=b \circ f_{1} \circ r$. Call the generated equivalence relation $\approx$.

Corollary 7. Let $f_{0}: X_{0} \rightarrow Y_{0}$ and $f_{1}: X_{1} \rightarrow Y_{1}$.

(1) $f_{0} \approx f_{1}$ iff $Y_{0}=Y_{1}$ and there exists a bomeomorphism $b:\left(C_{f_{0}}, X_{0}\right) \rightarrow$ $\left(C_{f_{1}}, X_{1}\right)$ rel $Y_{0}=Y_{1}$.

(2) $f_{0} \approx f_{1}$ iff there exists a homeomorphism $b:\left(C_{f_{0}} ; X_{0}, Y_{0}\right) \rightarrow$ $\left(C_{f_{1}} ; X_{1}, Y_{1}\right)$.

Proof. That the equivalence relation implies the corresponding mapping cylinder result goes through just as in Theorem 6 . The proofs the other way are obtained from Theorem 6. We will do the proof for (1). If $b$ is the homeomorphism of pairs rel $Y$, then one easily constructs a homeomorphism of $C_{f_{0}}$ and a mapping cylinder for $f_{1} \circ\left(b \mid X_{0}\right)$ which is rel $X_{0} \cup Y_{0}$. Hence, $f_{0} \sim f_{1} \circ$ $\left(b \mid X_{0}\right)$ and $f_{1} e \approx f_{1} \circ\left(b \mid X_{0}\right)$. So $f_{0} \approx f_{1}$.

From Corollary 7 and Proposition IV.10, we obtain a quick proof of an alternate formulation of Corollary V.3:

Corollary 8. Let $f_{0}: X_{0} \rightarrow Y_{0}$ and $f_{1}: X_{1} \rightarrow Y_{1}$ be p. l. maps with $f_{0} \approx f_{1}$. If $f_{0}$ is transverse cellular then $f_{1}$ is.

We now apply the mapping cylinder results to obtain a different uniqueness theorem for regular extensions. Recall that the uniqueness theorem Proposition V.4 part (2) involves varying the range by a transverse cellular map. Such variation can destroy nice properties of a map in general (see $\$$ VII) and it is desirable to have a theorem of the domain variation type. 
Proposition 9. Let $X_{0}$ be a subpolybedron of $X$ and $f: X_{0} \rightarrow Y_{0}$ be a p. l. map. Let $X \rightarrow{ }^{F^{1}} Y_{1} \leftarrow{ }^{i_{1}} Y_{0}$ and $X \rightarrow{ }^{F^{2}} Y_{2} \leftarrow{ }^{i_{2}} Y_{0}$ be regular extensions of $f$. Then $F_{1} \approx F_{2}$.

Proof. By Proposition V.4 part (2), we need only deal with the case where there exists a transverse cellular map $G: Y_{1} \rightarrow Y_{2}$ with $G^{-1}\left(i_{2}\left(Y_{0}\right)\right)=$ $i_{1}\left(Y_{0}\right), G \circ i_{1}=i_{2}$ and $F_{2}=G \circ F_{1}$. Triangulate $X, Y_{1}$ and $Y_{2}$ so that $X_{0}$, $i_{1}\left(Y_{0}\right)$ and $i_{2}\left(Y_{0}\right)$ are full subcomplexes and $F_{1}$ and $G$ are simplicial (and so $F_{2}$ is too). Choose deriveds so that everything is simplicial also.

Consider the simplicial map $C(\bar{G}): C_{F_{1}} \rightarrow C_{F_{2}}$.

By Proposition $1, C(\bar{G})$ is a regular extension. Hence, if $A$ is a simplex of $C_{F_{2}}-Y_{2}, D(A ; C(\bar{G}))$ is a cone on its boundary.

Now if $A \in Y_{2}-i_{2}\left(Y_{0}\right), D(A ; C(\bar{G})) \searrow D(A ; G)$ homogeneously in $C_{F_{1}}$ because $C(\bar{G})^{-1} \eta A=G^{-1} \eta A$; so III.2 and [C 2 , VII.10] apply. Since $G$ is transverse cellular we know that $D(A ; G)>$ point homogeneously in $Y_{1}$. But $D(A ; G) \subset\left|Y_{1}-i_{1}\left(Y_{0}\right)\right|$ and the inclusion $Y_{1} \subset C_{F_{1}}$ is locally collared on $\left|Y_{1}-i_{1}\left(Y_{0}\right)\right|$ so this collapse is homogeneous in $C_{F_{1}}$, too. So $D(A ; C(\bar{G}))$ is a cone on its boundary for $A \in Y_{2}-i_{2}\left(Y_{0}\right)$, also.

This leaves the crucial case $A \in i_{2}\left(Y_{0}\right)$. But since $C(\bar{G})^{-1} i_{2}\left(Y_{0}\right)=i_{1}\left(Y_{0}\right)$ and $C(\bar{G}) i_{1} \mid Y_{0}=i_{2} i_{1}^{-1}$, we have $(D, \dot{D})(A ; C(\bar{G}))=(D, \dot{D})\left(i_{1} i_{2}^{-1} A ; C_{F_{1}}\right)$, just as in part (1) of the proof of Lemma V.2.

Thus, $C(\bar{G})$ is a transverse cellular map of triads $\left(C_{F_{1}} ; \eta X, Y_{1}\right) \rightarrow$ $\left(C_{F_{2}} ; \eta X, Y_{2}\right)$. So we can obtain a homeomorphism

$$
\left(C_{F_{1}}, Y_{1}\right) \cong\left(C_{F_{2}}, Y_{2}\right) \text { rel } X .
$$

So by Corollary $7, F_{1} \approx F_{2}$.

VII. Transverse cellularity and homotopy. This section has its genesis in the error discovered by the referee.

Let $f_{0}, f_{1}: X \rightarrow Y$. Then we can define a relation $f_{0} \leftrightarrow_{e} f_{1}$ if there exist transverse cellular maps quasi-concordant to the identity: $d: X \rightarrow X$ and $r$ : $Y \rightarrow Y$ with $r f_{0}=f_{1} d$. Call the generated equivalence relation $\leftrightarrow$.

I originally "proved" Theorem VI.6 using $\leftrightarrow$ instead of $\sim$. However, this is false.

In fact, the following proposition is an almost antithetical result.

Proposition. Let $f_{0}, f_{1}: X \rightarrow Y$ be p. l. maps with $X$ and $Y$ compact. $f_{0} \leftrightarrow f_{1}$ if and only if $f_{0}$ is bomotopic to $f_{1}$.

Proof. $f_{0} \leftrightarrow f_{1}$ clearly implies homotopy so $\leftrightarrow$ does.

Conversely, if $f_{0}$ is homotopic to $f_{1}$ then there exists $f: X \times I \rightarrow Y \times I$ level-preserving, $f_{0}$ on 0 and $f_{1}$ on 1 . Triangulate so that the diagram $X \times I$ 
$\rightarrow f Y \times I \rightarrow^{\pi} \tau I$ is simplicial, and assume that the subdivision of $I, \tau I$, has vertices $t_{0}=0<t_{1}<\cdots<t_{n}=1$. Let $s_{i}=1 / 2\left(t_{i}+t_{i+1}\right), i=0, \cdots, n-1$. We claim $f_{s_{i}} \leftrightarrow e f_{t_{i}}$ and $f_{s_{i}} \leftrightarrow{ }_{e} f_{t_{i+1}}$. So $f_{0} \leftrightarrow f_{1}$.

Derive $X \times I, Y \times I, \tau I$ so that the above $f$ and $\pi$ diagram is still simplicial and $\eta\left[t_{i}, t_{i+1}\right]=s_{i}$. Then, $N\left(X \times t_{i} ; \eta\left(X \times\left[t_{i}, t_{i+1}\right]\right)\right)=X \times\left[t_{i}, s_{i}\right]$ with boundary $X \times s_{i}$. Let $T_{1}: N\left(X \times t_{i} ; \eta X \times\left[t_{i}, t_{i+1}\right]\right) \rightarrow X \times t_{i}$ and $T_{2}$ : $N\left(Y \times t_{i} ; \eta\left(Y \times\left[t_{i}, t_{i+1}\right]\right)\right) \rightarrow Y \times t_{i}$ be the natural retractions. Then we obtain the commutative diagram:

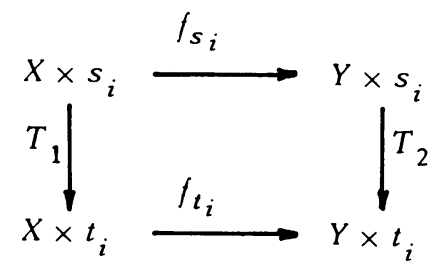

So $f_{s_{i}} \leftrightarrow_{e} f_{t_{i}}$ by Corollary VI.5. Similarly, $f_{s_{i}} \leftrightarrow_{e} f_{t_{i+1}}$.

VIII. Equalizer theorems. An application of transverse cellularity due in essence to Dancis is to the following conjecture. Given two embeddings $g_{0}$ and $g_{1}: X \rightarrow Y$ then a sufficient condition for them to be ambient isotopic should be the existence of a transverse cellular map $f: Y \rightarrow Z$ with $f_{g_{0}}=f_{g_{1}}$, i.e. an equalizer, e.g. if $g: X \times l \rightarrow M$ is an embedding into a manifold then let $f$ be a regular extension of $\pi_{X} \circ G^{-1}: G(X \times I) \rightarrow X$. This we generalize below. As stated this is not quite enough, for if $Y$ is a cone and $C$ is a regular neighborhood of the cone point of $Y$, and $X$ is a point then if $g_{0}$ and $g_{1}$ put the point anywhere in the interior of $C$, then a regular extension to $Y$ of $C \rightarrow$ point is an equalizer for $g_{0}$ and $g_{1}$, but, while isotopic, $g_{0}$ and $g_{1}$ need not be ambient isotopic.

The proper statement is the following:

Proposition 1. Let $g_{0}, g_{1}: X \rightarrow Y$ be embeddings such that there exists $f:$ $Y \rightarrow Z$ satisfying

(1) $f g_{0}=f g_{1}$ and is an embedding. Let $Z_{0}=f g_{i}(X)$.

(2) $f:\left(Y, g_{i}(X)\right) \rightarrow\left(Z, Z_{0}\right)$ is transverse cellular for $i=0,1$.

Then $g_{0}$ and $g_{1}$ are ambient is otopic.

Proof. By Proposition IV.2 there exist $\bar{f}_{i}: \quad Y \rightarrow Z$ homeomorphisms associated to $f$ with $\bar{f}_{i} g_{i}=f g_{i}, i=0,1$. So $\bar{f}_{1}^{-1} \bar{f}_{0}: Y \rightarrow Y$ is a homeomorphism ambient isotopic to the identity taking $g_{0}$ to $g_{1}$. Both $\bar{f}_{i}$, s can be assumed carried by the same isomorphism of cone complexes from $X$ to $Y$, and hence the is otopy result.

A corollary is closer to the original theorem of Dancis.

Corollary 2. Let $g_{0}, g_{1}: X \rightarrow Y$ be embeddings such that there exists $Y \supset$ 
$Y_{0} \supset g_{0}(X) \cup g_{1}(X)$ and $f: Y_{0} \rightarrow Z$ satisfying

(1) $f g_{0}=f g_{1}$ and is a bomeomorphism of $X$ with $Z$.

(2) $f^{-1}(x) \searrow f^{-1}(x) \cap g_{i}(x)$ bomogeneously in $Y$ for $x \in Z$ and $i=0,1$.

Then $g_{0}$ and $g_{1}$ are ambient isotopic.

Proof. Any regular extension of $f$ satisfies the conditions of Proposition 1, see Corollary IV.4.

Remark. In applying Corollary 2, we note that Lemma IV.7 implies that $f^{-1}(x){ }_{b} \searrow f^{-1}(x) \cap g_{i}(x), i=0,1$, iff $f^{-1}(x)$ collapses homogeneously to some point and

$$
d\left(f^{-1}(x) \cap g_{i}(x) ; Y\right)=\min \left\{d(y ; Y): y \in f^{-1}(x)\right\}, \quad i=0,1 .
$$

Corollary 3. Let $G: X \times I \rightarrow Y$ be an embedding such that for each $x \in X$, $d(G(x, t) ; Y)$ is constant for $t$ varying in $[0,1]$, then the embeddings $g_{0}$ and $g_{1}$ are ambient isotopic.

Proof. In Corollary 2, let $Y_{0}=G(X \times I)$ and $f=$ proj $\circ G^{-1}: G(X \times I) \rightarrow X$.

IX. Fiberwise transversality. Once upon a time, Dancis asked me if one could prove a level-preserving version of Cohen's theorems on transverse cellularity. It was this question which started me on this work and so it is satisfying to be able to include an affirmative answer.

Lemma 1. Let $X$ be a compact polybedron and let $X \rightarrow^{\pi} I$ be a p. l. map such that for each subinterval $J$ of $I$, the inclusion $\pi^{-1}(j) \subset \pi^{-1}(J)$ is locally collared, then there exists a bomeomorphism $b: \pi^{-1}(0) \times I \rightarrow X$ rel $\pi^{-1}(0)$ with $\pi b(x, t)=t$, i.e. $b$ preserves levels.

Proof. A simple variation of the Hudson-Zeeman trick is used to cover isotopies by covering them locally.

First, we define pieces of $b$, locally. If $J \subset I$ (subinterval) and $a \in j$, then by collaring there exists a homeomorphism $\left(a \in J^{\prime} \subset J\right)$ :

$$
k: \pi^{-1}(a) \times J^{\prime} \cong V \operatorname{rel} \pi^{-1}(a)
$$

where $V$ is a regular neighborhood of $\pi^{-1}(a)$ in $\pi^{-1}(J)$.

Triangulate the diagram

$$
\pi^{-1}(a) \times J^{\prime} \stackrel{k}{\longrightarrow} \pi^{-1}\left(J^{\prime}\right) \stackrel{\pi}{\longrightarrow} J^{\prime}
$$

and choosing $J_{0}$ a small enough subinterval of $J^{\prime}=[a, b]$, such that if $v$ is a vertex of $\pi^{-1} J_{0}$ or $k^{-1} \pi^{-1} J_{0}$ then $\pi v=a$ or $\pi k v=a$. Then we can change $k$ so that it is level-preserving on $\pi^{-1}(a) \times J_{0}$, following the Hudson-Zeeman technique of $[\mathrm{H}, 6.7]$.

Thus, for each $t$ in $I$, we can find a neighborhood $J_{t}$ of $t$ in $I$, and a 
home omorphism $k_{t}: \pi^{-1}(t) \times J_{t} \rightarrow \pi^{-1}\left(J_{t}\right)$ level-preserving and rel $\pi^{-1}(t)$. To construct the global homeomorphism we use the compactness of $I$ analogous to the Hudson-Zeeman method for covering isotopies (see [H, 6.12.2]). We can find a sequence $0=t_{0}<t_{1}<\cdots<t_{n}=1$ such that $t_{i+1} \in J_{t_{i}}$ for $0 \leq i<n$. Inductively we define $H_{i+1}: \pi^{-1}(0) \times\left[0, t_{i+1}\right] \rightarrow \pi^{-1}\left[0, t_{i+1}\right]$ to be equal to $H_{i}$ on $\pi^{-1}(0) \times\left[0, t_{1}\right]$ and by letting $H_{i+1}(x, t)$ equal $k_{t_{i}}\left(p_{1}\left(k_{t_{i}}^{-1} H_{i}\left(x, t_{i}\right)\right), t\right)$ for $(x, t) \epsilon$ $\pi^{-1}(0) \times\left[t_{i}, t_{i+1}\right]$. Then $b=H_{n}$.

Proposition 2. Let $f: K \times I \rightarrow L \times I$ be a level-preserving, transverse cellular simplicial map of finite complexes with $L \times I$ triangulated so that the projection $\pi: L \times I \rightarrow \sigma l$ is simplicial for some subdivision $\sigma l$.

There exists a level-preserving homeomorphism $g: K \times I \rightarrow L \times I$ carried by $\gamma_{f}$, and if $T$ is a subcomplex of $\sigma$ l such that $f \mid K \times T$ is a bomeomorphism then $g$ can be taken equal to $f$ on $K \times T$.

Proof. By starting at 0 and proceeding up the one-simplices of $\sigma l$ we reduce the situation to a one-simplex $J \in \sigma l$. So we must show that if $f: K \times J \rightarrow L \times$ $J \rightarrow^{\pi} J$ is simplicial we can find $g: K \times J \cong L \times J$, where if $f$ is a homeomorphism on $K \times a$ or $K \times b$ or both $([a, b]=J)$ then $g$ can be chosen to agree with $f$ there.

For $A \in L \times a, \dot{D}(A ; L \times J)=\bigcup\{D(B ; L \times J): A<B \in L \times a\} \cup C(A ; L \times J)$ where (definition) $C(A ; L \times J) \equiv \bigcup\{D(B ; L \times J): A<B \in L \times J-L \times a\}$. $C(A ; L \times J)$ is a cone on $\bigcup\{C(B ; L \times J): A<B \in L \times a\}$ because it is the complement of a regular neighborhood of $\dot{D}(A ; L \times a)$ in $\dot{D}(A ; L \times J)$, see $\left[\mathrm{C}_{2}, 4.2\right]$.

$f$ preserves this decomposition, i.e. $\dot{D}(A ; f)=\bigcup\{D(B ; f): A<B \in L \times a\} \cup$ $C(A ; f)$ where $C(A ; f) \equiv \bigcup\{D(B ; f): A<B \in L \times J-L \times a\}$.

Now $f \mid C(A ; f): C(A ; f) \rightarrow C(A ; L \times J)$ is transverse cellular since $f \mid D(B ; f)$ is for each $B \in L \times J$. Since $f_{a}$ is transverse cellular (Corollary V.10), $f: D\left(A ; f_{a}\right) \rightarrow D(A ; L \times a)$ is also. Hence

$$
\begin{aligned}
\left(D(\dot{A ; f}) ; C(A ; f), D\left(A ; f_{a}\right)\right) & \cong(D(A ; L \times J) ; C(A ; L \times J), D(A ; L \times a)) \\
& \cong((D(A ; L \times a) \times I ; D(A ; L \times a) \times 1, D(A ; L \times a) \times 0))
\end{aligned}
$$

(for the latter homeomorphism see $\left[C_{2}, 4.2\right.$ proof $]$ or $[A, 6.2$ proof $]$ ).

Now $\pi f: D(A ; f) \rightarrow[a, \eta J]$ and $\pi: D(A ; L) \rightarrow[a, \eta J]$ are simplicial maps. So $(\pi f)^{-1}[a, t]$ in $D(A ; f)$ is a regular neighborhood of $D\left(A ; f_{a}\right)$ with boundary $\pi f^{-1}(t)$ and hence $\pi f^{-1}(t)$ is collared on both sides. Similarly, for $\pi$ : $D(A ; L) \rightarrow[a, \eta J]$.

Thus, both $\pi f: D(A ; f) \rightarrow[a, \eta J]$ and $\pi: D(A ; L) \rightarrow[a, \eta J]$ satisfy the hypotheses of Lemma 1 . Thus for each $A \in L \times a$, there are level-preserving home omorphisms: 


$$
\begin{aligned}
k_{A}: D\left(A ; f_{a}\right) \times[a, \eta J] & \cong D(A ; f) \quad \text { rel } D\left(A ; f_{a}\right) \times a=D\left(A ; f_{a}\right), \\
l_{A}: D(A ; L \times a) \times[a, \eta J] & \cong D(A ; L) \quad \text { rel } D(A ; L \times a) \times a=D(A ; L \times a),
\end{aligned}
$$

and similarly for each $A \in L \times b$ we get a homeomorphism involving the interval $[\eta J, b]$. by

Now choose any homeomorphism $g: K \times\{a, \eta J, b\} \rightarrow L \times\{a, \eta J, b\}$, carried

$$
\begin{aligned}
& D\left(A ; f_{a}\right) \rightarrow D(A ; L \times a) \text { for } A \in L \times a, \\
& D(B ; f) \rightarrow D(B ; L \times J) \text { for } B \in L \times J-L \times j, \\
& D\left(A ; f_{b}\right) \rightarrow D(A ; L \times b) \text { for } A \in L \times b .
\end{aligned}
$$

Choose $g$ equal to $f$ on any piece where $f$ is already a homeomorphism.

Note that $g(C(A ; f))=C(A ; L \times J)$ for either $A \in L \times a$ or $A \in L \times b$.

By induction we can extend $g$ in a level-preserving way over $K \times J$. If $g$ : $\dot{D}(A ; f) \cup D\left(A ; f_{a}\right) \cong \dot{D}(A ; L \times J) \cup D(A ; L \times a)$ is defined either level-preserving for $\underset{A}{A} \in L \times a$, ther

$$
\begin{aligned}
l_{A}^{-1} g k_{A}:\left(\dot{D}\left(A ; f_{a}\right)\right. & \times[a, \eta J]) \cup\left(D\left(A ; f_{a}\right) \times\{a, \eta J\}\right) \\
& \rightarrow(\dot{D}(A ; L \times a) \times[a, \eta J]) \cup(D(A ; L \times a) \times\{a, \eta J\})
\end{aligned}
$$

is a level-preserving homeomorphism. We can cone this map using the Alexander trick and extend $l_{A}^{-1} g k_{A}$ to a level-preserving homeomorphism on $D\left(A ; f_{a}\right) \times$ $[a, \eta J]$. Composing with $l_{A}$ and $k_{A}^{-1}$ extends $g$ as required.

As a corollary we can sharpen Proposition IV.15 to analogous results concerning quasi-isotopies, in the compact case.

Corollary 3. (1) Two bomeomorphisms are quasi-isotopic iff they are isotopic.

(2) Two bomeomorphisms associated to a transverse cellular map are isotopic.

(3) A bomeomorphism is quasi-isotopic to a transverse cellular map iff it is isotopic to an associated homeomorphism.

(4) Transverse cellular maps are quasi-isotopic iff associated homeomorphis ms are isotopic.

It seems reasonable that Proposition 2 could be generalized by replacing $I$ by $\Delta^{n}$, i.e.

Conjecture. Let $K \times \Delta^{n} \rightarrow^{f} L \times \Delta^{n} \rightarrow^{\pi} \Delta^{n}$ be simplicial with $f$ transverse cellular level-preserving. Then there exists a level-preserving homeomorphism $g: K \times \Delta^{n} \rightarrow L \times \Delta^{n}$ carried by $\gamma_{f}$ and such that if $J$ is a union of faces of $\Delta^{n}$ such that $f \mid K \times J$ is a homeomorphism then $g$ can be chosen to equal $f$ on $K \times J$.

A consequence of this conjecture, by induction on the simplices of $M$, would be the same statement with $M$ replacing $\Delta^{n}$.

Another corollary of this would be the following: 
Conjecture. Let $p_{1}: E_{1} \rightarrow B$ and $p_{2}: E_{2} \rightarrow B$ be locally trivial p. 1. maps, and let $f: E_{1} \rightarrow E_{2}$ be a fiber-preserving transverse cellular map. Then for any $\epsilon>0$ there exists a fiber-preserving homeomorphism $g: E_{1} \rightarrow E_{2}$ within $\epsilon$ of $f$ (for any metric on $E_{2}$ ).

This would follow by triangulating $B$ so that $E_{1}$ and $E_{2}$ are trivial over each simplex of $B$, and then subdivided so that the mesh of $E_{1}$ and $E_{2}$ are small and $E_{1} \rightarrow{ }^{f} E_{2} \rightarrow^{p_{2}} B$ is simplicial (hence $p_{1}: E_{1} \rightarrow B$ is). Proceed by induction on the simplices of $B$.

This result seems approachable in another manner. For $A \in B$ let $E_{i}^{A}=$ $p_{i}^{-1} A$. Then $f:\left(E_{1} ; E_{1}^{A}: A \in B\right) \rightarrow\left(E_{2} ; E_{2}^{A}: A \in B\right)$ is transverse cellular as a map of families. So by family version of Proposition IV.1, we can find $g$ : $\left(E_{1} ; E_{1}^{A}: A \in B\right) \cong\left(E_{2} ; E_{2}^{A}: A \in B\right)$. It is then a matter of changing $g$ inductively to be level-preserving. Thus we need something like $g: F \times \Delta \rightarrow F \times \Delta$ levelpreserving on $F \times \dot{\Delta}$ can be changed to a level-preserving homeomorphism.

Also, it should be noted that this family argument applies directly to blockbundles, i.e. a block preserving transverse cellular map can be changed to a blockbundle isomorphism.

\section{B IBLIOGRA PHY}

[A] E. Akin, Manifold phenomena in the theory of polyhedra, Trans. Amer. Math. Soc. 143 (1969), 413-473. MR 40 \#6544.

$\left[\mathrm{C}_{1}\right]$ M. M. Cohen, Simplicial structures and transverse ce!lularity, Ann. of Math. (2) 85 (1967), 218-245. MR $35 \# 1037$.

$\left[\mathrm{C}_{2}\right] \longrightarrow$, A general the ory of relative regular neighborhoods, Trans. Amer. Math. Soc. 136 (1969), 189-229. MR 40 \#2052.

$\left[\mathrm{C}_{3}\right]-$ Homeomorphisms between homotopy manifolds and their resolutions, Invent. Math. 10 (1970), 239-250.

[D] J. Dancis, Unpublished corre spondence.

[H] J. F. P. Hudson, Piecewise linear topology, Benjamin, New York, 1969. MR $40 \# 2094$.

[L] W. B. R. Lickorish, The piecewise linear unknotting of cones, Topology 4 (1965), 67-91. MR $34 \# 3585$.

DEPARTMENT OF MATHEMATICS, THE CITY COLLEGE, NEW YORK, NEW YORK 10031 